\title{
Uncertainty and Preference Modelling for Multiple Criteria Vehicle Evaluation
}

\author{
Xinlian Xie* \\ Transportation Management College, Dalian Maritime University \\ Dalian, 116026, China \\ Manchester Business School, The University of Manchester \\ Manchester, M15 6PB, United Kingdom \\ *Email:xxlian77@yahoo.com \\ Jian-Bo Yang and Dong-Ling Xu \\ Manchester Business School, The University of Manchester \\ Manchester, M15 6PB, United Kingdom \\ Anil Kumar Maddulapalli \\ India Science Lab, GM R\&D, Bangalore, India \\ Qiuping Yang \\ Transportation Management College, Dalian Maritime University \\ Dalian, 116026, China \\ Received: 12-08-2009 \\ Accepted: 27-10-2010
}

\begin{abstract}
A general framework for vehicle assessment is proposed based on both mass survey information and the evidential reasoning (ER) approach. Several methods for uncertainty and preference modeling are developed within the framework, including the measurement of uncertainty caused by missing information, the estimation of missing information in original surveys, the use of nonlinear functions for data mapping, and the use of nonlinear functions as utility function to combine distributed assessments into a single index. The results of the investigation show that various measures can be used to represent the different preferences of decision makers towards the same feedback from respondents. Based on the ER approach, credible and informative analysis can be conducted through the complete understanding of the assessment problem in question and the full exploration of available information.
\end{abstract}

Keywords: Multiple criteria analysis; Vehicle evaluation; Uncertainty modeling; Evidence theory

\section{Introduction}

Uncertainty is one of the main concerns in most decision making processes and has been researched intensively ${ }^{1-6}$. Although the types and sources of uncertainty for various problems may be different, they share some common features ${ }^{7}$. As an empirical study, this paper is aimed to investigate pragmatic ways for handling uncertainty in assessment of vehicle attributes.

Evaluating vehicle attributes is an important activity of a process which can help both OEMs (i.e., original equipment manufacturers) and consumers understand the quality and attractiveness of various vehicles. To facilitate vehicle attribute assessment, different types of surveys and tests may be conducted for gathering firsthand data. These surveys and tests might be conducted internally by an OEM, independent specialist consultancy companies, consumer groups, or government departments.

One example is the survey carried out by J.D. Power and Associates, Automotive Performance Execution And Layout Study (APEAL). It is a mail survey that asks the consumers what they like and don't like about the various vehicle attributes listed on the questionnaire. It measures customer ratings on retail purchasers and lessees from randomly drawn samples on over 100 vehicle attributes including ride and handling, engine and transmission, comfort and convenience and so on ${ }^{8}$. 
In APEAL, vehicle attributes are rated using a ten point scale on which 1 might represent 'unacceptable' while 10 might represent 'outstanding'. To make the data as accessible as possible, experts at J. D. Power convert thousands of questions and answers into scores in a few categories, which are then aggregated to give vehicle attributes an overall score. This pivotal figure is then expressed as a percentage and an overall rating? ${ }^{9}$.

Another example is the rating listed in the Consumer Reports published by Consumers Union ${ }^{10-12}$. Consumer Reports rates the performance of the vehicles tested by the experts of Consumers Union at its specialized autotest facility and compiles regularly updated charts showing which models perform best and worst overall. Assessments of vehicles on many designated attributes are based on various scales among which an ordinal 5point scale may take the following form:

- 1 = "Poor"

- 2 = "Fair"

- 3 = "Good",

- 4 = "Very Good"

- $5=$ "Excellent"

The examples show that to distinguish or rank vehicle attributes, more than one rating or survey on vehicles may be obtained (especially for big OEMs who might even have their own surveys on vehicles). It is obvious that each survey has its own style and specific attribute settings. The statements used in one survey may somewhat differ from those used in other surveys, although they may belong to the same or similar attributes.

It is often the case that an analyst needs to use multiple surveys in assessing vehicle attributes. Hence, it is important to combine assessments from diverse surveys in order to produce comprehensive and consistent assessments. This requires transformation or mapping of survey data from various formats to a common format. While linear functions are most common and might perform well for certain transformations, a question arises as to whether nonlinear transformation functions might perform better in other cases. This forms one of the research questions investigated in this paper. It is likely that a subject (consumer or expert assessor) may not provide assessments for a vehicle on certain attributes. It is also possible that in a hierarchy of detailed vehicle attributes, there may be partial or no assessments on certain attributes. Such missing information can cause problems in follow-up analysis and need to be handled properly in order to provide reliable and non-distorted overall assessments on vehicles. This paper is devoted to investigating these issues for objective and consistent vehicle attribute assessment based on multiple surveys.

In this paper, a general framework for vehicle attribute assessment is firstly constructed on the basis of the Evidential Reasoning (ER) approach. The procedure for constructing and using this general framework in assessment of vehicle attributes is discussed in Section 2. In Section 3, various methods for estimating missing information in surveys are investigated. Sections 4 and 5 are devoted to studying the impacts of nonlinear mapping functions and utility functions on overall assessments. The application of these methods for dealing with uncertainty is demonstrated using a case study in Section 6, and the paper is concluded in Section 7.

\section{The General Assessment Framework and Related Issues}

The information propagation and aggregation in assessing vehicle attributes can be conducted using the Evidential Reasoning (ER) approach ${ }^{13}$. Based on different survey results or original ratings, the comprehensive assessments or overall ratings of vehicle attributes can be generated using the IDS Multi-criteria Assessor software ${ }^{14}$, which has been developed on the basis of the ER approach ${ }^{15}$. The ER approach and the IDS software provide a methodological basis and a tool for this research.

As stated in Section 1, multiple surveys, different forms of questionnaires and various rating scales may be used for assessing vehicle attributes. It is usually desirable to take into account as much evidence as available and appropriate for vehicle assessment, including imprecise and incomplete information that may exist in surveys. Next, we propose and discuss a generic procedure for assessing and ranking vehicle attributes under the above-mentioned scenario.

\subsection{Proposed generic procedure}

The proposed generic procedure includes six main steps as follows. 
(1) Collect and validate data from all surveys for all related vehicles.

(2) Construct a detailed vehicle attribute hierarchy.

(3) Construct an attribute assessment hierarchy based on evidence aggregation logic and algorithms.

(4) Transform survey data to assessments measured on a common scale using mapping functions.

(5) Use the IDS software to aggregate all transformed assessments for a vehicle attribute.

(6) Rank vehicle attributes based on overall assessments.

These steps are normally followed in the above order, though there may be interactions between these steps in the process of vehicle assessment. For example, the order between step (3) and step (4) does not have to be followed strictly. Next, we discuss a general hierarchy structure for assessing different attributes of a vehicle.

\subsection{General hierarchy structure for attribute assessment}

Fig. 1 illustrates a general hierarchy for assessing vehicle attributes. A vehicle attribute might have information from multiple surveys and might have more than one related statement within a survey. Different criteria might be used to relate survey data to a vehicle attribute and hence assess that particular vehicle attribute. For example, a criterion could be that the mean rating for a survey statement of an OEM's vehicle should be greater than the mean rating for that survey statement of a competitor OEM's vehicle. The hierarchy incorporates sub-criteria within a criterion and the hierarchy could be expanded both horizontally and vertically. The ER approach, discussed in Subsection 2.4, is capable of handling complex hierarchies. The survey data is usually collected at the leaf node of a branch and then the ER approach is used to aggregate the data bottom-up for each vehicle attribute.

As mentioned above, different surveys use different scales for obtaining ratings of the statements in the questionnaire. For aggregating survey information related to vehicle attributes, survey data have to be transformed to a common scale. In the next sub-section, we discuss a representative common scale that can be used for assessing vehicle attributes.

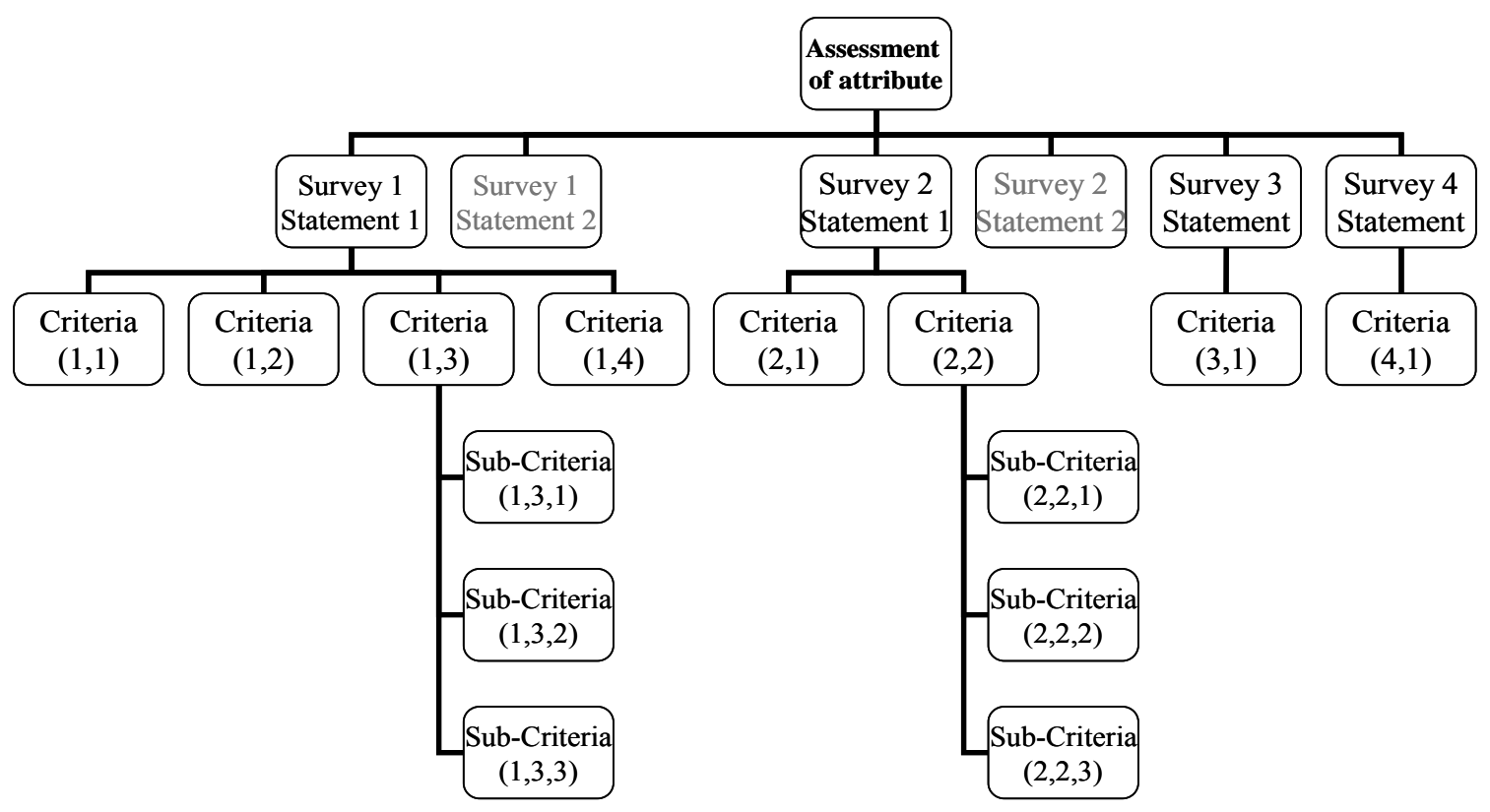

Fig. 1. A general hierarchical structure for attribute assessment

\subsection{The common scale}

Assessments on an attribute in various surveys may be measured on different scales, which may be judgmental, cardinal, or ordinal. Also, a scale may have various grades. As such, a common scale is employed in the general assessment framework, so that all assessments generated from various surveys can be measured on the same scale and direct comparisons among the 
assessments can be made. For example, a viable common scale with an ordinal five grades from 1 to 5 is listed below. To take into account missing information, one more grade (unassigned priority) can be added to the common scale.

- $5=$ "Top priority"

- 4 = "High priority"

- 3 = "Average priority"

- 2 = "Low priority"

- $1=$ "No priority"

- $\mathrm{UN}=$ "Unassigned priority"

We use the above common scale for illustrating our results in the coming sections.

\subsection{The Evidential Reasoning algorithm and its logic}

The ER algorithm is developed on the basis of a multiattribute evaluation framework ${ }^{16}$ and the evidence combination rule of the Dempster-Shafer (D-S) theory ${ }^{17}$. It can be used to aggregate criteria of a multilevel structure. It is employed in the general assessment framework for propagating and aggregating information. The rationale and logic of the ER approach has been discussed in Refs. 15 and 18-20.

The main function of the ER algorithm in the general assessment framework is to calculate the combined degrees of belief of a group of criteria for generating an assessment on their parent criterion using a group of recursive formulas. Such calculations are conducted from the lowest level to the top level in an assessment hierarchy as shown in Fig. 1, and eventually the overall assessment can be obtained in terms of a distributed assessment. To assist in ranking a series of vehicle attributes, the concept of expected utility may be used as an auxiliary measure.

A general-purpose multiple criteria decision analysis tool, the Intelligent Decision System (IDS) software ${ }^{14}$ can be used for this purpose, which is based on the Evidential Reasoning (ER) approach. IDS provides Windows-based graphical interfaces to support the building of decision models where all vehicle attributes can be assessed on a general hierarchy using a belief structure. The rank order of all vehicle attributes can be generated on the basis of utility scores. With the help of the IDS software, the general assessment framework can be employed efficiently and easily.
Among the advantages of using this framework for assessing vehicle attributes, the explicit representation and propagation of uncertainty in the information aggregation process is of particular interest. By allowing imprecise and incomplete information to be modeled for assessment of vehicle attributes, the framework can be widely applicable and can produce transparent and reliable results in a consistent and transparent way. Imprecise and incomplete information in the assessment of vehicle attributes may be incurred from missing information in an original survey, from data transformation and representation, and so on. It is crucial to measure and express all types of uncertainty properly before the process of assessing vehicle attributes is started.

As such, three related issues will be studied in more detail in the following sections. First, the measurement of uncertainty caused due to missing information in original surveys is studied. The estimation of missing information is also discussed for certain circumstances. Secondly, the possibility and properties of using nonlinear functions for data transformation are investigated. Thirdly, the properties of using nonlinear functions as utility function in aggregating distributed assessments into a single index are studied for the cross comparison and rank ordering of attributes.

The skewness of evaluation rating may also be a source for uncertainty in both data survey and transformation stages. This type of uncertainty can be dealt with by using nonlinear functions as mapping function or as utility function in some circumstances as discussed in Sections 4 and 5.

\section{Missing Information}

\subsection{Measurement of uncertainty}

Missing information often exists in a survey. There are basically two types of missing information in a survey. One is failed observation, which means that there is no valid information from a respondent. This may be because the respondent has no idea or simply refuses to give his or her opinion about all survey statements. The other is failed data or missing information in a valid observation, which means that a respondent does give valid responses to at least one, but not all, questions in a questionnaire. This might be because the respondent only has part of the knowledge required to answer the 
full questionnaire or the questionnaire is time consuming to complete. In the former situation, the failed observation either can be taken as no information and be counted in the sample or can simply be deleted and not counted in the sample. In the latter situation, the observation with failed data or missing information should be taken into account as it is normal for a respondent to have only partial knowledge about a specific vehicle but still have interests to participate in the survey and provide what he or she knows. Note that we assume that the respondent does not deliberately (or by any other means) give incorrect response when he or she has the knowledge about the vehicle.

For a single vehicle attribute, the total number of valid observations is the sample size, and the number of observations which have no valid response is the amount of missing information. The amount of missing information is divided by the sample size to give the level of missing information or unassigned belief degree which is defined as the measure of uncertainty in question. Based on this definition, uncertainty caused due to missing information can be determined by Eq. (1) as follows.

$$
U N=\frac{T_{s}-M_{s}}{T_{s}}
$$

where $U N$ - unassigned belief degree or the measure of uncertainty

$T_{s}$ - sample size for evaluation of a vehicle attribute

$M_{s}$ - number of respondents who provide valid response for evaluation of a vehicle attribute.

Sometimes criteria used for relating survey data to a vehicle attribute might need information from two or more vehicles. For example, relative position of two vehicles in a particular survey could be used for assessing the importance of an attribute. In such cases, Eq. (2) can be used for taking into account the impact of missing information for both vehicles. Note that Eq. (2) can be easily extended for taking into account data from more than two vehicles.

$$
U N=\varphi_{s 1} \cdot \frac{T_{s 1}-M_{s 1}}{T_{s 1}}+\varphi_{s 2} \cdot \frac{T_{s 2}-M_{s 2}}{T_{s 2}}
$$

where $U N$ - unassigned belief degree or the measure of uncertainty for comparison of two vehicles

$T_{s 1}$ — sample size for the first vehicle

$T_{s 2}$ - sample size for the second vehicle

$M_{s 1}$ - number of respondents who provide valid response for the first vehicle
$M_{s 2}$ - number of respondents who provide valid response for the second vehicle

$\varphi_{s 1}, \varphi_{s 2}$ - the respective weights for the two vehicles in determining uncertainty, and $\varphi_{s 1}+\varphi_{s 2}=1$.

One can set $\varphi_{s 1}=\varphi_{s 2}=\frac{1}{2}$ if the impact of individual vehicle's sample size is not to be accounted for or can set $\varphi_{s 1}=\frac{T_{s 1}}{T_{s 1}+T_{s 2}}$ and $\varphi_{s 2}=\frac{T_{s 2}}{T_{s 1}+T_{s 2}}$ if the impact of individual sample sizes is to be accounted for. Other methods of setting the weights $\varphi_{s 1}$ and $\varphi_{s 2}$ might be used according to individual circumstances and the preferences of the analyst.

For example, if the sample size and the number of valid responses in a sample for an attribute of the first vehicle are 200 and 192 respectively, the level of missing information or the uncertainty in the evaluation on that attribute is 0.04 by Eq. (1). If we set $\varphi_{s 1}=\varphi_{s 2}=\frac{1}{2}$ and the sample size and the number of valid responses for the same attribute of the second vehicle are 240 and 228, the level of missing information or the uncertainty in the evaluation comparison of the two vehicles on the attribute is 0.045 by Eq. (2).

A special case in Eq. (2) that needs to be noted is that $T_{s 1}$ or $T_{s 2}$ or both are equal to 1 . If $T_{s 1}$ is equal to 1 and $M_{s 1}$ is equal to 0 or $T_{s 2}$ is equal to 1 and $M_{s 2}$ is equal to 0 , the uncertainty degree calculated using Eq. (2) may not be equal to 1 though it should be so. As in this case there must be no valid response for one of the two vehicles, the value of uncertainty obtained using Eq. (2) does not make sense if $U N \neq 1$. In fact, if $M_{s 1}$ or $M_{s 2}$ is less than a certain threshold value, the corresponding $U N$ should be artificially assigned to 1, which means that the comparison between the two vehicles is improper if there is a lack of valid information.

\subsection{Accommodating uncertainty in questionnaire}

In a large scale survey, it is normal to have some subjects who might not have complete knowledge about the survey or can not provide full confidence in some assessments. To accommodate such a scenario, we propose below a new form of questionnaire that would give more freedom and flexibility to the respondents and could result in the collection of original and better quality data. 
In the proposed questionnaire, the uncertainty in the subjects' response is explicitly captured by allowing the subject to provide a distributed assessment as shown in Table 1. An overall assessment on the attribute can then be calculated by Eq. (3) and Table 2.

$$
B I_{n}=\sum_{j=1}^{m} \zeta_{j} \cdot B I C B_{n, j}
$$

where $B I_{n}$ - the mean belief degree on grade $n$ $(n=1, \cdots, N)$ of an attribute

$m$ - total number of respondents,

$B I C B_{n, j}$ - belief degree on grade $n$ for an attribute given by respondent $j$,

$\zeta_{j}$ — the weight of the $j^{\text {th }}$ respondent in evaluating an attribute, and $\sum_{j=1}^{m} \zeta_{j}=1$.
Typically, if all respondents are given the same importance, $\zeta_{j}=\frac{1}{m}$. Table 1 takes a traditional survey format as a special case, where a respondent ticks only one box (with 100\% degree of belief), and allows more flexible yet realistic answers to survey questions. The above-suggested format of questionnaire is only one example out of many possibilities. It can be designed to be more user-friendly but without losing its essential characteristics.

\subsection{Estimation of missing information}

In this subsection, we discuss some cases in which missing information on an attribute can be estimated as a function of related information on other attributes. We propose some functions for estimating the missing information in those cases.

Table 1 Proposed model to account for uncertainty in assessment

\begin{tabular}{|c|c|c|c|c|c|c|}
\hline \multicolumn{7}{|l|}{ Survey Statement } \\
\hline Assessment grade & Worst (1) & Poor (2) & Average (3) & Good (4) & Excellent (5) & Unsure (6) \\
\hline Degree of belief & & & & & & \\
\hline
\end{tabular}

Table 2 Aggregation of assessments on a survey statement's rating

\begin{tabular}{|c|l|c|c|}
\hline Grade & \multicolumn{1}{|c|}{ Description } & Belief degree (\%) & Remark \\
\hline 1 & Worst & $B I_{1}$ & \\
\hline 2 & Poor & $B I_{2}$ & \\
\hline 3 & Average & $B I_{3}$ & \\
\hline 4 & Good & $B I_{4}$ & \\
\hline 5 & Excellent & $B I_{5}$ & \\
\hline 6 & Uncertainty & $B I_{6}$ & \\
\hline \multicolumn{2}{|c|}{ Total belief degree } & & $0 \leq$ and $\leq 100 \%$ \\
\hline
\end{tabular}

In a survey, there are often many attributes to be assessed which may be grouped in a hierarchy. There might also be cases where a set of attributes from various surveys are related in one way or other. In vehicle evaluation, for example, independent survey providers may disclose their survey results and OEMs may have their own evaluations, although statements for a similar attribute may be different in such various surveys. If such relationships can be explicitly expressed in a hierarchy, the following approach can be used for estimating missing information from surveys.

Suppose an attribute $A$ can be expressed fully by a set of sub-attributes $a_{i}, i=1,2, \ldots, k$, and $k \geq 2$. Then, the attribute $A$ is dependent on its sub-attributes which are assumed to be mutually independent for assessment. Suppose assessments on attributes provided in survey(s) include missing information. It is also assumed that the same scale is used in the assessments. If different evaluation scales are used, assessments given on various scales can be transformed to a common scale using the mapping functions discussed in Section 4.

Depending on the characters of missing information there could be six different cases to consider. The first case is one where all assessments are given. This is an ideal case and does not require any estimation of missing information. The second case is one where neither a parent attribute nor its sub-attributes are assessed. In this case, there might be no need or it might 
be inappropriate to estimate missing information simply because of the complete lack of information. The other four cases are discussed in detail below.

Case 1 - Assessment on the high level (or parent) attribute is unknown, but all assessments on its subattributes are known.

Eq. (4) can be used to estimate the unknown assessment of attribute $A$.

$$
A t_{j}=\sum_{i=1}^{k} \omega_{i} \cdot a v_{i, j} \quad j=1,2, \ldots \ldots, N
$$

where $A t_{j}$ - estimated assessment value on the $j^{\text {th }}$ grade of the attribute $A$

$\omega_{i}$ - weight of the $i^{\text {th }}$ sub-attribute in the assessment on its parent attribute, and $\sum_{i=1}^{k} \omega_{i}=1$

$a v_{i, j}$ - assessment value on the $j^{\text {th }}$ grade of the $i^{\text {th }}$ subattribute $a_{i}$

$k$ - number of sub-attributes related to the same parent attribute $A$

$N$ - number of grades on the assessment scale

In this case, the estimated assessment on $A$ is of full confidence as long as the assessments on $a_{i}, i=1,2, \ldots$, $k$ are of full confidence. More precisely, the estimated assessment on $A$ determined by Eq. (4) has the same confidence degree as the sub-attributes.

Case 2 - Assessment on the parent attribute is unknown, and some assessments on its sub-attributes are known.

If there are $s$ out of $k$ sub-attributes with unknown assessments, there will be obviously $s$ degrees of freedom in estimating the unknown assessments. In other words, $s$ conditions are needed to estimate the unknown assessments. If $s$ conditions indeed exist and can be identified, the estimation of the unknown assessments can be uniquely made with full confidence. Otherwise, necessary conditions have to be subjectively established and the confidence of the estimation may vary according to the reliability of the subjective conditions established.

For example, if the weights of the related subattributes can be generated by a fuzzy AHP procedure ${ }^{21}$, the weighted average of known assessments can be used as the estimation of the unknown assessments. Among a variety of ways for establishing the necessary conditions, the following Eq. (5) is a simple and convenient one.

$$
a v_{k_{1}, j}=a v_{k_{2}, j}=\cdots=a v_{k_{s}, j}=\frac{1}{\left|I_{2}\right|} \sum_{i_{2} \in I_{2}} a v_{i_{2}, j}
$$

$$
k_{1}, k_{2}, \ldots \ldots, k_{s} \in I_{1}
$$

where $I_{1}$ - subscript set for the sub-attributes with unknown assessments

$I_{2}$ - subscript set for the sub-attributes with known assessments

$\left|I_{2}\right|$ - number of sub-attributes in $I_{2}$

$a v_{i_{2}, j}-$ known assessment on the $j^{\text {th }}$ grade of sub-attribute $i_{2}$

With $s$ given conditions, the assessment on the unknown attribute $A$ can be made using Eq. (6).

$$
\begin{gathered}
A t_{j}=\sum_{i_{2} \in I_{2}} \omega_{i_{2}} \cdot a v_{i_{2}, j}+\sum_{i_{1} \in I_{1}} \omega_{i_{1}} \cdot a v_{i_{1}, j} \\
j=1,2, \ldots \ldots, N
\end{gathered}
$$

It is hard to determine the confidence of the estimation given by Eq. (6), if $s \neq 0$. However for each estimate, under certain conditions, it is possible to determine an interval within which the true value might exist. As the value of each grade $a v_{i, j}$ is defined in the closed interval $[0,1]$, Eq. (7) gives the maximum estimate of $A t_{j}$ by setting all $a v_{i_{1}, j}=a v_{i_{1} j}^{\max }=1$ for $i_{1} \in I_{1}$, and Eq. (8) gives the minimum estimate of $A t_{j}$ by setting all $a v_{i_{1}, j}=0$ for $i_{1} \in I_{1}$, respectively.

$$
\begin{gathered}
A t_{j}^{\max }=\sum_{i_{2} \in I_{2}}^{i_{i_{1}, j}} \omega_{i_{2}} \cdot a v_{i_{2}, j}+\sum_{i_{1} \in I_{1}} \omega_{i_{1}} \cdot a v_{i_{1}, j}^{\max } \\
A t_{j}^{\min }=\sum_{i_{2} \in I_{2}}^{j} \omega_{i_{2}} \cdot a v_{i_{2}, j}+\sum_{i_{1} \in I_{1}} \omega_{i_{1}} \cdot a v_{i_{1}, j}^{\min } \\
j=1,2, \ldots \ldots, N
\end{gathered}
$$

where $A t_{j}^{\max }$ - maximum estimate of $A t_{j}$ $A t_{j}^{\mathrm{min}}$ - minimum estimate of $A t_{j}$

At the extreme points of $a v_{i_{1} j}^{\max }=1$ and $a v_{i_{1}, j}^{\min }=0$, we have,

$$
\begin{gathered}
A t_{j}^{\max }=\sum_{i_{2} \in I_{2}} \omega_{i_{2}} \cdot a v_{i_{2}, j}+\sum_{i_{1} \in I_{1}} \omega_{i_{1}} \\
j=1,2, \ldots \ldots, N \\
A t_{j}^{\min }=\sum_{i_{2} \in I_{2}} \omega_{i_{2}} \cdot a v_{i_{2}, j} \\
j=1,2, \ldots \ldots, N
\end{gathered}
$$

Therefore, we have the estimation interval [ $\left.A t_{j}^{\min }, A t_{j}^{\max }\right]$ for $A t_{j}$ with confidence. Alternatively, we have the following relationship:

$$
0 \leq A t_{j}^{\min } \leq A t_{j} \leq A t_{j}^{\max } \leq 1
$$

In a similar way and based on Eqs. (7) and (8), the estimation interval for each unknown sub-attribute can be deduced to give Eqs. (9) and (10) respectively. 


$$
\begin{gathered}
a v_{k_{h}, j}^{\max }=\min \left[1, \frac{1}{\omega_{k_{h}}}\left(A t_{j}^{\max }-\sum_{i_{2} \in I_{2}} \omega_{i_{2}} \cdot a v_{i_{2}, j}\right)\right] \\
j=1,2, \ldots \ldots, N \\
a v_{k_{h}, j}^{\min }=\max \left[0, \frac{1}{\omega_{k_{h}}}\left(A t_{j}^{\min }-\sum_{i_{2} \in I_{2}} \omega_{i_{2}} \cdot a v_{i_{2}, j}-\sum_{\substack{i_{1} \in I_{1} \\
i_{1} \neq k_{h}}} \omega_{i_{1}}\right)\right] \\
j=1,2, \ldots \ldots, N \\
k_{h} \in I_{1} \text { or } 1 \leq h \leq s
\end{gathered}
$$

where $a v_{k_{h}, j}^{\max }$ - maximum estimate of $a v_{k_{h}, j}$

$a v_{k_{h}, j}^{\min }$ - minimum estimate of $a v_{k_{h}, j}$

For given $s$ and $s \geq 1$, we can select any unknown sub-attribute and determine its estimation interval using Eqs. (7) and (8) or (9) and (10), depending on whether or not the selected sub-attribute has sub-attributes of its own or not. Then, based on the same rationale and the estimation interval of the first estimated sub-attribute, the estimation interval for the second unknown sub-attribute can be determined using Eqs. (7) and (8) or (9) and (10) again. This process can be repeated until the estimation intervals for the $s+1$ (i.e., $s$ sub-attributes and one parent attribute) unknown attributes are obtained.

For example, suppose a parent attribute $A$ has six sub-attributes $a_{i}, i=1,2, \ldots, 6$ and the sub-attributes from $a_{1}$ to $a_{4}$ have complete assessment information as shown in Table 3, while the remaining two sub-attributes have unknown assessments. The estimated values and the estimation intervals of assessments for the last two sub-attributes and the parent attribute are calculated using Eqs. (5) to (10) and are listed in Table 3. As the estimation intervals for all the grades of the two unknown sub-attributes are outside the rational interval $[0,1]$ of the grade definition, the applicable estimation intervals need to be adjusted into the closed interval $[0,1]$.

It is interesting to note that in the tests we conducted, the applicable estimation intervals, after adjustment, for all the sub-attributes are equal to the complete closed interval $[0,1$,$] . This is in fact the nature of Eqs. (9) and$ (10). Therefore, Eqs. (9) and (10) or to guess the estimation interval of an unknown sub-attribute is actually meaningless in this case. However, the estimation interval of the unknown parent attribute is relatively small and stable. In many cases, the estimation interval for the parent attribute is of importance and is used for determining the final assessment interval of a detailed vehicle attribute or the vehicle itself under uncertainty.

Case 3 - Assessment on the parent attribute is known, and some assessments on its sub-attributes are unknown.

If there are $s$ out of $k$ sub-attributes with unknown assessments, there will obviously be $(s-1)$ degrees of freedom in estimating unknown assessments. If $(s-1)$ conditions are met, the unknown assessments can be estimated with confidence. Otherwise, necessary conditions have to be subjectively established and the

\begin{tabular}{|c|c|c|c|c|c|c|c|}
\hline Attribute & Grade 1 & Grade 2 & Grade 3 & Grade 4 & Grade 5 & $\omega$ & Remark \\
\hline At (value) & 0.0175 & 0.1475 & 0.33 & 0.4125 & 0.0925 & & \multirow{2}{*}{ Predicted } \\
\hline At (interval) & {$[0.01,0.31]$} & {$[0.11,0.41]$} & {$[0.24,0.54]$} & {$[0.27,0.57]$} & {$[0.07,0.37]$} & & \\
\hline$a_{1}$ & 0.1 & 0.2 & 0.6 & 0.1 & 0 & 0.1 & known \\
\hline$a_{2}$ & 0 & 0 & 0 & 0.8 & 0.2 & 0.2 & known \\
\hline$a_{3}$ & 0 & 0.3 & 0.6 & 0 & 0.1 & 0.3 & known \\
\hline$a_{4}$ & 0 & 0 & 0 & 1 & 0 & 0.1 & known \\
\hline$a_{5}$ (value) & 0.025 & 0.125 & 0.3 & 0.475 & 0.075 & \multirow{2}{*}{0.05} & \multirow{2}{*}{ Predicted } \\
\hline$a_{5}$ (interval) & {$[0,1]$} & {$[0,1]$} & {$[0,1]$} & {$[0,1]$} & {$[0,1]$} & & \\
\hline$a_{6}$ (value) & 0.025 & 0.125 & 0.3 & 0.475 & 0.075 & \multirow{2}{*}{0.25} & \multirow{2}{*}{ Predicted } \\
\hline$a_{6}$ (interval) & {$[0,1]$} & {$[0,1]$} & {$[0,1]$} & {$[0,1]$} & {$[0,1]$} & & \\
\hline
\end{tabular}
confidence of the estimation obtained under these conditions may vary according to the reliability of the subjective conditions established.

Table 3 The maximum and minimum values of missing assessments 
For example, if we assume that each grade value of the unknown sub-attributes are equally correlated to the grade values of the parent attribute and the known subattributes, and

$$
\begin{gathered}
a v_{k_{1}, j}=a v_{k_{2}, j}=\cdots=a v_{k_{s}, j} \\
k_{1}, k_{2}, \ldots \ldots, k_{s} \in I_{1}
\end{gathered}
$$

Eq. (12) can be used to estimate the grade values of the unknown sub-attributes.

$$
a v_{k_{h}, j}=\left(A t_{j}-\sum_{i_{2} \in I_{2}} \omega_{i_{2}} \cdot a v_{i_{2}, j}\right) / \sum_{i_{1} \in I_{1}} \omega_{i_{1}}
$$

Eq. (6) is used to pair with the ( $s-1)$ given conditions expressed in Eq. (11) to form $s$ equations. The estimation intervals of these $s$ unknown sub-attributes can be determined using Eqs. (9) and (10) in which both $A t_{j}^{\min }$ and $A t_{j}^{\max }$ should be substituted by $A t_{j}$. The parent attribute with six sub-attributes as discussed in Case 2 is used to demonstrate the estimation of the two unknown sub-attributes. At this time, suppose the assessment on the parent attribute is known as shown in Table 4. As both $A t_{j}^{\min }$ and $A t_{j}^{\max }$ are substituted by $A t_{j}$ in Eqs. (9) and (10), the estimation intervals for $a_{5}$

\begin{tabular}{|c|c|c|c|c|c|c|c|}
\hline Attribute & Grade 1 & Grade 2 & Grade 3 & Grade 4 & Grade 5 & $\omega$ & Remark \\
\hline$A t$ & 0.0175 & 0.1475 & 0.33 & 0.4125 & 0.0925 & & known \\
\hline$a_{1}$ & 0.1 & 0.2 & 0.6 & 0.1 & 0 & 0.1 & known \\
\hline$a_{2}$ & 0 & 0 & 0 & 0.8 & 0.2 & 0.2 & known \\
\hline$a_{3}$ & 0 & 0.3 & 0.6 & 0 & 0.1 & 0.3 & known \\
\hline$a_{4}$ & 0 & 0 & 0 & 1 & 0 & 0.1 & known \\
\hline$a_{5}$ (value) & 0.025 & 0.125 & 0.3 & 0.475 & 0.075 & \multirow{2}{*}{0.05} & \multirow{2}{*}{ Predicted } \\
\hline$a_{5}$ (interval) & {$[0,0.15]$} & {$[0,0.75]$} & {$[0,1]$} & {$[0,1]$} & {$[0,0.45]$} & & \\
\hline$a_{6}$ (value) & 0.025 & 0.125 & 0.3 & 0.475 & 0.075 & \multirow{2}{*}{0.25} & \multirow{2}{*}{ Predicted } \\
\hline$a_{6}$ (interval) & {$[0,0.03]$} & {$[0,0.15]$} & {$[0.16,0.36]$} & {$[0.37,0.57]$} & {$[0,0.09]$} & & \\
\hline
\end{tabular}
and $a_{6}$ are reduced to a smaller range after adjustment or deleting void area produced by Eqs. (9) and (10).

Table 4 The estimation values of missing assessments for two sub-attributes

Case 4 - Assessment on the parent attribute is known, but all the assessments on its sub-attributes are unknown.

Generally speaking, for any given sub-attribute $h$, its unknown assessments can be determined by Eq. (13), assuming that all other sub-attributes are guessed in advance.

$$
\begin{aligned}
& a v_{h, j}=\frac{1}{\omega_{h}}\left(A t_{j}-\sum_{\substack{i=1 \\
i \neq h}}^{k} \omega_{i} \cdot a v_{i, j}\right) \\
& h=1,2, \ldots, k ; j=1,2, \ldots \ldots, N
\end{aligned}
$$

Similar to Case 3 , if we assume that each grade value of the unknown sub-attributes is equally correlated to the grade values of the parent attribute and Eq. (11) is used as the necessary $(k-1)$ conditions, Eq.(14) can be used to estimate the grade values of the unknown sub-attributes..

$$
\begin{gathered}
a v_{h, j}=A t_{j} \\
h=1,2, \ldots, k ; j=1,2, \ldots \ldots, N
\end{gathered}
$$

To obtain the maximum estimation value of $a v_{h, j}$, let all other $a v_{i, j}=0, i=1,2, \ldots, k$ and $i \neq h$ in Eq. (13). Then, we have

$$
\begin{gathered}
a v_{h, j}^{\max }=\min \left[1, \frac{A t_{j}}{\omega_{h}}\right] \\
h=1,2, \ldots, k ; j=1,2, \ldots, N
\end{gathered}
$$

Similarly, to obtain the minimum estimation value of $a v_{h, j}$, let all other $a v_{i, j}=1, i=1,2, \ldots, k$ and $i \neq h$. Eq.(13) gives

$$
\begin{aligned}
a v_{h, j}^{\min } & =\max \left[0, \frac{1}{\omega_{h}}\left(A t_{j}-\sum_{\substack{i=1 \\
i \neq h}}^{k} \omega_{i}\right)\right] \\
h & =1,2, \ldots, k ; j=1,2, \ldots, N
\end{aligned}
$$

To demonstrate Eqs. (14) - (16), the example employed in Case 2 and Case 3 is again used for Case 4 with only assessment for the parent attribute being given as shown in Table 5. It is interesting to find that the applicable estimation intervals after adjustment are likely to fall into a closed interval that is smaller than $[0$, $1]$. 
Table 5 The estimation values of missing assessments for all sub-attributes

\begin{tabular}{|c|c|c|c|c|c|c|c|}
\hline Attribute & Grade 1 & Grade 2 & Grade 3 & Grade 4 & Grade 5 & $\omega$ & Remark \\
\hline$A t$ & 0.0175 & 0.1475 & 0.33 & 0.4125 & 0.0925 & & known \\
\hline$a_{1}$ (value) & 0.018 & 0.148 & 0.330 & 0.413 & 0.093 & \multirow{2}{*}{0.1} & \multirow{2}{*}{ Predicted } \\
\hline$a_{1}$ (interval) & {$[0,0.175]$} & {$[0,1]$} & {$[0,1]$} & {$[0,1]$} & {$[0,0.925]$} & & \\
\hline$a_{2}$ (value) & 0.018 & 0.148 & 0.330 & 0.413 & 0.093 & \multirow{2}{*}{0.2} & \multirow{2}{*}{ Predicted } \\
\hline$a_{2}$ (interval) & {$[0,0.088]$} & {$[0,0.738]$} & {$[0,1]$} & {$[0,1]$} & {$[0,0.463]$} & & \\
\hline$a_{3}($ value $)$ & 0.018 & 0.148 & 0.330 & 0.413 & 0.093 & \multirow{2}{*}{0.3} & \multirow{2}{*}{ Predicted } \\
\hline$a_{3}$ (interval) & {$[0,0.058]$} & {$[0,0.492]$} & {$[0,1]$} & {$[0,1]$} & {$[0,0.308]$} & & \\
\hline$a_{4}($ value $)$ & 0.018 & 0.148 & 0.330 & 0.413 & 0.093 & \multirow{2}{*}{0.1} & \multirow{2}{*}{ Predicted } \\
\hline$a_{4}$ (interval) & {$[0,0.175]$} & {$[0,1]$} & {$[0,1]$} & {$[0,1]$} & {$[0,0.925]$} & & \\
\hline$a_{5}$ (value) & 0.018 & 0.148 & 0.330 & 0.413 & 0.093 & \multirow{2}{*}{0.05} & \multirow{2}{*}{ Predicted } \\
\hline$a_{5}$ (interval) & {$[0,0.35]$} & {$[0,1]$} & {$[0,1]$} & {$[0,1]$} & {$[0,1]$} & & \\
\hline$a_{6}$ (value) & 0.018 & 0.148 & 0.330 & 0.413 & 0.093 & \multirow{2}{*}{0.25} & \multirow{2}{*}{ Predicted } \\
\hline$a_{6}$ (interval) & {$[0,0.07]$} & {$[0,0.59]$} & {$[0,1]$} & {$[0,1]$} & {$[0,0.37]$} & & \\
\hline
\end{tabular}

Except for Case 1, there might be multiple solutions for all the other three cases. Additional conditions or constraints are therefore needed for finding a specific solution in each of these three cases. In Eqs. (5) to (16), we assumed that all unknown assessments are equal. This is the simplest yet viable assumption for estimating missing information and it is most likely to generate the mean values for missing assessments. Without doubt, if more dedicated conditions can be established under specific circumstances, better estimates for unknown assessments can be generated with confidence.

In the next section, we investigate and discuss the affects of non-linear mapping functions for transforming survey data on to the common scale.

\section{Mapping Function}

As discussed in previous section, in many circumstances, there is a need for transforming assessments from an original survey to a desired scale which may differ from the evaluation scale used in the original survey. Linear mapping functions such as the one shown in Fig. 2 are the simplest ones. However, there are also various nonlinear functions that could be used for data transformation. To demonstrate some properties of nonlinear functions for data transformation, a quadratic function $f(x)=a x^{2}+b x+c$ is taken as an example in this section.

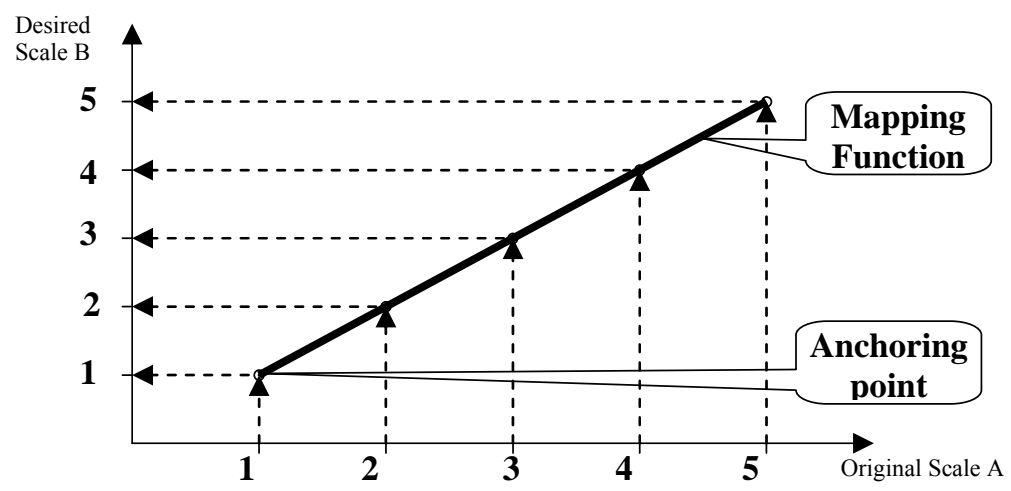

Fig. 2. Linear mapping from one scale to another 
In a quadratic function, there could be at most three unknown parameters $a, b$ and $c$ and hence three conditions are needed to determine a quadratic function. For simplicity, we assume that the anchoring point and the top rank point of a mapping function are the same in original scale A and desired scale B. In other words, the lowest grade in the original scale A for a survey is mapped to the lowest grade in the desired scale B and the highest grade in the original scale $\mathrm{A}$ is mapped to the highest grade in the desired scale B, as shown in Fig. 2. Furthermore, we assume that the middle point of assessment on the original scale is mapped into $t$ on the desired scale. Based on these assumptions, the following relationship can be established.

$$
f(x, t)=a(t) x^{2}+b(t) x+c(t)
$$

where $x$ - independent variable which stands for the assessment grade on the original scale

$t$ - value of $f\left(x_{m}, t\right)$ at the middle point $x_{m}$ of the original scale, which is a parameter used to control the shape of the parabola

$f(x, t)$ - assessment on the desired scale corresponding to $x$, given the parameter $t$

Assuming that the original scale A and the desired scale B have five assessment grades as shown in Fig. 2, the values for the parameters in Eq. (17) would then be:

$$
a(t)=\frac{3-t}{4}, b(t)=\frac{3 t-7}{2}, c(t)=\frac{15-5 t}{4} .
$$

Fig. 3 shows a set of parabolas following Eq. (17) with $x_{m}=3$ as the middle point in the original scale. Note that, if $t=3$, then $a=0$ and $c=0$, and $f(x)$ becomes a straight line or a linear function as shown in Fig. 3. If $t<3$, the grades between the lowest and highest grades on the desired scale are devaluated in comparison with the corresponding grades on the original scale. If $t>3$, the grades between the lowest and highest grades on the desired scale are appreciated in comparison with the corresponding grades on an original scale.

The extents of devaluation or appreciation are quite different for different grades between the lowest and highest grades. These properties can be used to reduce or enlarge the range of assessments. For example, suppose that the original evaluations for six attributes are as shown in Table 6. A number in a column under a grade is the belief degree of the grade assessed for the corresponding attribute. The mean grade in the last column for an attribute is the average of grade numbers (i.e., 1 for the lowest grade and 5 for the highest grade) multiplied by corresponding belief degrees.

Take $t=2$ for example. $a(t)=0.25, b(t)=-0.5$ and $c(t)=1.25$, and the mapping function becomes a convex curve. The five grades 1, 2, 3, 4 and 5 on the original scale are mapped to $1,1.25,2,3.25$ and 5 respectively on the desired scale. To find the belief degrees of an assessment on each grade on the desired scale, piecewise linear approach can be employed.

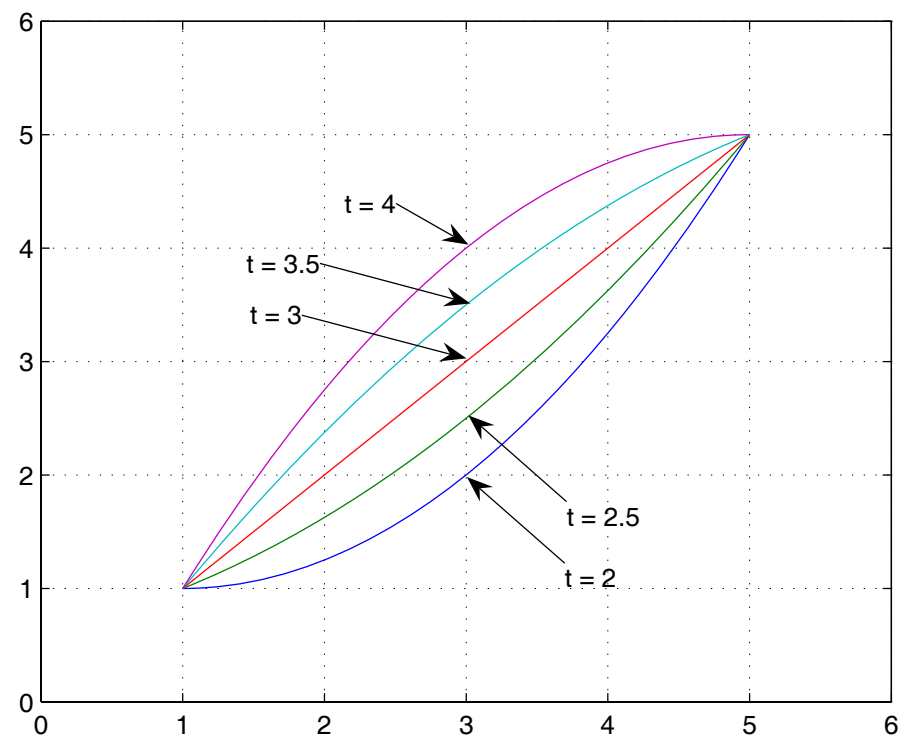

Fig. 3. Parabolas of the quadratic function with different value for parameter $t$ 
If a grade on the original scale coincides with a grade on the desired scale after mapping, then the belief degree of the grade for an attribute on the original scale is directly transformed to the grade on the desired scale. Otherwise, if a grade on the original scale moves to a place in between two adjacent grades, say Grade $h$ and Grade $h+1$, on the desired scale after mapping, then the belief degree of the grade for an attribute on the original scale is transformed to the grades on the desired scale in the following way.

Suppose that $B o$ represent the belief degree of a grade for an attribute on the original scale before mapping. The grade on the original scale is mapped to a place $G d$ between Grade $h$ and Grade $h+1$ on the desired scale. Then,

$$
\begin{gathered}
B d_{h}=B o \times\left(1-\frac{G d-G d_{h}}{G d_{h+1}-G d_{h}}\right) \\
B d_{h+1}=B o \times \frac{G d-G d_{h}}{G d_{h+1}-G d_{h}}
\end{gathered}
$$

where: $B d_{h}, B d_{h+1}$ - belief degrees of Grade $h$ and $h+1$ for an attribute on the desired scale respectively after mapping
$G d_{h}, G d_{h+1}$ - scale values of Grade $h$ and $h+1$ on the desired scale respectively

$G d$ - scale values between Grade $h$ and Grade $h+1$ on the desired scale after mapping, which is mapped from the grade on the original scale

After executing above calculations for all belief degrees of assessments on an attribute, the belief degrees of assessments on each grade on the desired scale for the same attribute should be added up to give the total that is the data in each cell in Table 7.

As expected in the example of data mapping from Table 6 to Table 7, after transformation, all the ratings are devaluated or shifted towards lower grades. The distribution range of the mean grades over the six attributes is also changed from [3.6, 4] on the original scale to $[2.85,3.4625]$ on the desired scale after mapping. It means that the distribution of the average ratings has been dispersed by the mapping function.

However, it is noticeable that the rank order of the six attributes based on the mean grades has changed after mapping in this example. Therefore, only when a nonlinear mapping function truly reflects a desired evaluation transformation, this approach can be used.

Table 6 Assessments on the original scale

\begin{tabular}{|c|c|c|c|c|c|c|}
\hline Attribute & Grade 1 & Grade 2 & Grade 3 & Grade 4 & Grade 5 & Mean \\
\hline$a_{1}$ & 0 & 0 & 0.6 & 0.2 & 0.2 & 3.6 \\
\hline$a_{2}$ & 0 & 0.2 & 0.2 & 0.3 & 0.3 & 3.7 \\
\hline$a_{3}$ & 0 & 0 & 0.2 & 0.8 & 0 & 3.8 \\
\hline$a_{4}$ & 0 & 0.1 & 0.1 & 0.7 & 0.1 & 3.8 \\
\hline$a_{5}$ & 0 & 0.15 & 0.15 & 0.3 & 0.4 & 3.95 \\
\hline$a_{6}$ & 0 & 0 & 0 & 1 & 0 & 4 \\
\hline
\end{tabular}

Table 7 Assessments on the desired scale using quadratic mapping function with $t=2$

\begin{tabular}{|c|c|c|c|c|c|c|}
\hline Attribute & Grade 1 & Grade 2 & Grade 3 & Grade 4 & Grade 5 & Mean \\
\hline$a_{1}$ & 0 & 0.6 & 0.15 & 0.05 & 0.2 & 2.85 \\
\hline$a_{2}$ & 0.15 & 0.25 & 0.225 & 0.075 & 0.3 & 3.125 \\
\hline$a_{3}$ & 0 & 0.2 & 0.6 & 0.2 & 0 & 3 \\
\hline$a_{4}$ & 0.075 & 0.125 & 0.525 & 0.175 & 0.1 & 3.1 \\
\hline$a_{5}$ & 0.1125 & 0.1875 & 0.225 & 0.075 & 0.4 & 3.4625 \\
\hline$a_{6}$ & 0 & 0 & 0.75 & 0.25 & 0 & 3.25 \\
\hline
\end{tabular}

To use a nonlinear mapping function without altering the rank order of a group of alternatives, the nonlinear mapping function should be applied to the mean grades instead of every individual grades. For 
example, if the same quadratic function with $t=2$ is used to map the mean grades of the six attributes listed in Table 6 , the rank order of their mean grades after mapping will be the same as the ranking before mapping. The mean grades before and after mapping are listed in the two columns under Example 1 in Table 8, which shows that the distribution interval for the mean grades is changed from $[3.6,4]$ to $[2.69,3.25]$. To further demonstrate this property of non-linear function, a ten attribute example, named Example 2, is also given in Table 8. By comparison of the two columns under Example 2, the distribution interval is changed from $[3.03,4]$ to $[2.03,3.25]$. However, the relative rankings of the attributes are not changed in both examples.

Table 8 Mean grade transformation using nonlinear mapping function

\begin{tabular}{|c|c|c|c|c|}
\hline \multirow{2}{*}{ Alternative } & \multicolumn{2}{|c|}{ Example 1 } & \multicolumn{2}{c|}{ Example 2 } \\
\cline { 2 - 5 } & $\begin{array}{c}\text { Mean grade before } \\
\text { mapping }\end{array}$ & $\begin{array}{c}\text { Mean grade after } \\
\text { mapping }\end{array}$ & $\begin{array}{c}\text { Mean grade before } \\
\text { mapping }\end{array}$ & $\begin{array}{c}\text { Mean grade after } \\
\text { mapping }\end{array}$ \\
\hline$a_{1}$ & 3.6 & 2.69 & 3.03 & 2.0302 \\
\hline$a_{2}$ & 3.7 & 2.8225 & 3.08 & 2.0816 \\
\hline$a_{3}$ & 3.8 & 2.96 & 3.19 & 2.1990 \\
\hline$a_{4}$ & 3.8 & 2.96 & 3.26 & 2.2769 \\
\hline$a_{5}$ & 3.95 & 3.1756 & 3.3 & 2.3225 \\
\hline$a_{6}$ & 4 & 3.25 & 3.36 & 2.3924 \\
\hline$a_{7}$ & & & 3.4 & 2.44 \\
\hline$a_{8}$ & & & 3.5 & 2.5625 \\
\hline$a_{9}$ & & & 3.59 & 2.6770 \\
\hline$a_{10}$ & {$[3.6,4]$} & {$[2.69,3.25]$} & $43.03,4]$ & {$[2.03,3.25]$} \\
\hline Interval & & &
\end{tabular}

These examples show that a nonlinear function used to transform evaluation data plays an important part in changing the distribution range. This property can be used to change the distribution pattern of assessments to a desirable one. Apart from the quadratic function discussed in this section, other types of nonlinear functions can also be used as mapping function. Some properties of the cubic function will be discussed in the next section. It is important to choose an appropriate nonlinear function as the mapping function. Such a choice is domain specific and requires expert knowledge.

\section{Utility Function}

The concept of expected utility has been introduced in the ER approach for rank ordering when the distributed overall assessments are not sufficient to show differences in ranking. As an auxiliary measure, utility is applied to the general vehicle assessment framework to help intuitively rank the detailed attributes or vehicles. Suppose $u\left(H_{j}\right)$ is the utility of the grade $H_{j}$ with

$$
u\left(H_{j+1}\right)>u\left(H_{j}\right) \quad \text { if } H_{j+1} \text { is preferred to } H_{j}
$$

If all assessments are complete and precise, the expected utility of an alternative $A$ can be calculated by

$$
u(A)=\sum_{j=1}^{N} \beta_{j} u\left(H_{j}\right)
$$

where $H_{j}-j^{\text {th }}$ grade on an evaluation scale, $j=1$, $2, \ldots, N$

$\beta_{j}$ - belief degree evaluated on grade $H_{j}$

$u(A)$ - expected utility of the alternative $A$.

$u\left(H_{j}\right)$ may be estimated using probability assignment methods $^{22-23}$ or by constructing regression models using partial rankings or pairwise comparisons ${ }^{20}$. In most cases, a linear function of $u\left(H_{j}\right)$ may be preferred because of its simplicity, although a nonlinear function may also be used to calculate utility in certain circumstances. It should be noted, however, that a utility function is used to capture the decision maker's preferences and as such it should be constructed using preference information provided by the decision maker. In this section, a cubic function is used to demonstrate the features of nonlinear functions in dealing with uncertainty and preference in assessment propagation and aggregation. A general cubic function can be expressed as follows.

$$
f(x)=a x^{3}+b x^{2}+c x+d
$$

As there are four unknown parameters $a, b, c$ and $d$ in Eq. (20), four conditions need to be given to 
determine the cubic function. Among various ways of giving the four conditions, for illustration purpose, fixing the middle point and the two ends of the definition interval for the utility function is a straightaway selection. The fourth condition could be determination of the slope at any one of the known points so that the shape of the cubic function inside the definition interval can be controlled explicitly.

In a general way, let $\left(x_{l}, y_{l}\right),\left(x_{h}, y_{h}\right)$ and $\left(x_{m}, y_{m}\right)$ be the starting, ending and middle points, respectively, in the definition interval of the utility function. In this example the slope of the utility function at the middle point is supposed to be given. Let $y=f(x)$, and $\frac{d y}{d x}=s$ at $x=x_{m}$. The four unknown parameters $a, b, c$ and $d$ can be determined by Eqs. (21) to (24).

$a=\frac{A\left(y_{m}-y_{h}\right)-C\left(x_{m}^{2}-x_{h}^{2}\right)-s A\left(x_{m}-x_{h}\right)+2 C x_{m}\left(x_{m}-x_{h}\right)}{A\left(x_{m}^{3}-x_{h}^{3}\right)-B\left(x_{m}^{2}-x_{h}^{2}\right)-3 A x_{m}^{2}\left(x_{m}-x_{h}\right)+2 B x_{m}\left(x_{m}-x_{h}\right)}$

$$
b=\frac{C-B a}{A}
$$

$$
\begin{gathered}
c=s-3 x_{m}^{2} a-2 x_{m} b \\
d=y_{h}-a x_{h}^{3}-x_{h}^{2} b-x_{h} c
\end{gathered}
$$

where $A=2 x_{m}\left(x_{l}-x_{m}\right)-\left(x_{l}^{2}-x_{m}^{2}\right)$

$$
\begin{aligned}
& B=3 x_{m}^{2}\left(x_{l}-x_{m}\right)-\left(x_{l}^{3}-x_{m}^{3}\right) \\
& C=s\left(x_{l}-x_{m}\right)-\left(y_{l}-y_{m}\right)
\end{aligned}
$$

For example, suppose the values for the three known points be given by

$$
\begin{aligned}
& x_{l}=1, y_{l}=0 ; \\
& x_{h}=5, y_{h}=1 ; \\
& x_{m}=3, y_{m}=0.5 .
\end{aligned}
$$

The distinct shapes of the cubic function in the definition interval $[1,5]$ are shown in Fig. 4 with different slope values at the middle point. In Fig. 4, line $y 1$ corresponds to $s_{1}=0, y 2$ to $s_{2}=1 / 5, y 3$ to $s_{3}=1 / 4, y 4$ to $s_{4}=1 / 3$ and $y 5$ to $s_{5}=1 / 2$. Fig. 5 describes the slope change along the transverse axis $x$ of the cubic functions in the definition interval.

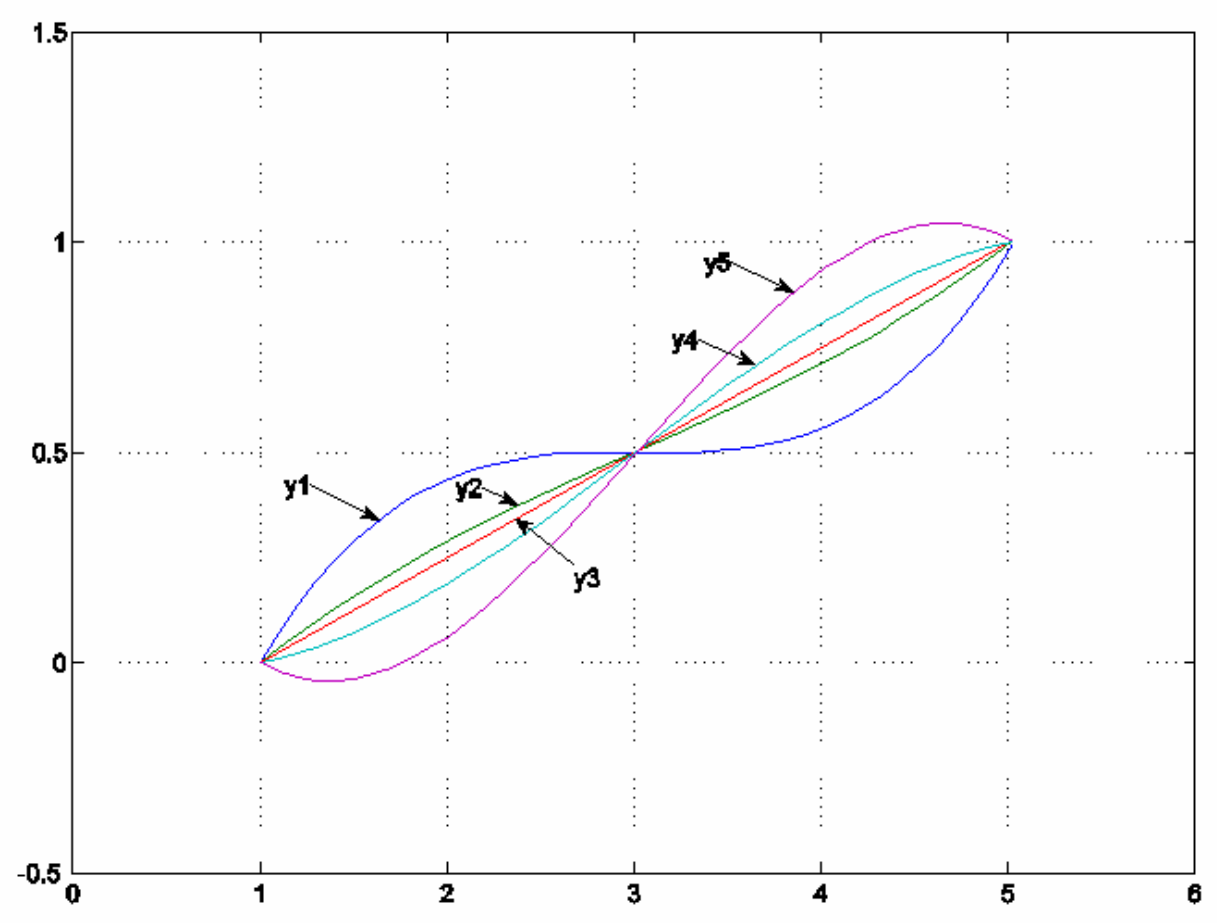

Fig. 4. Cubic utility functions with varying slopes at the middle point 


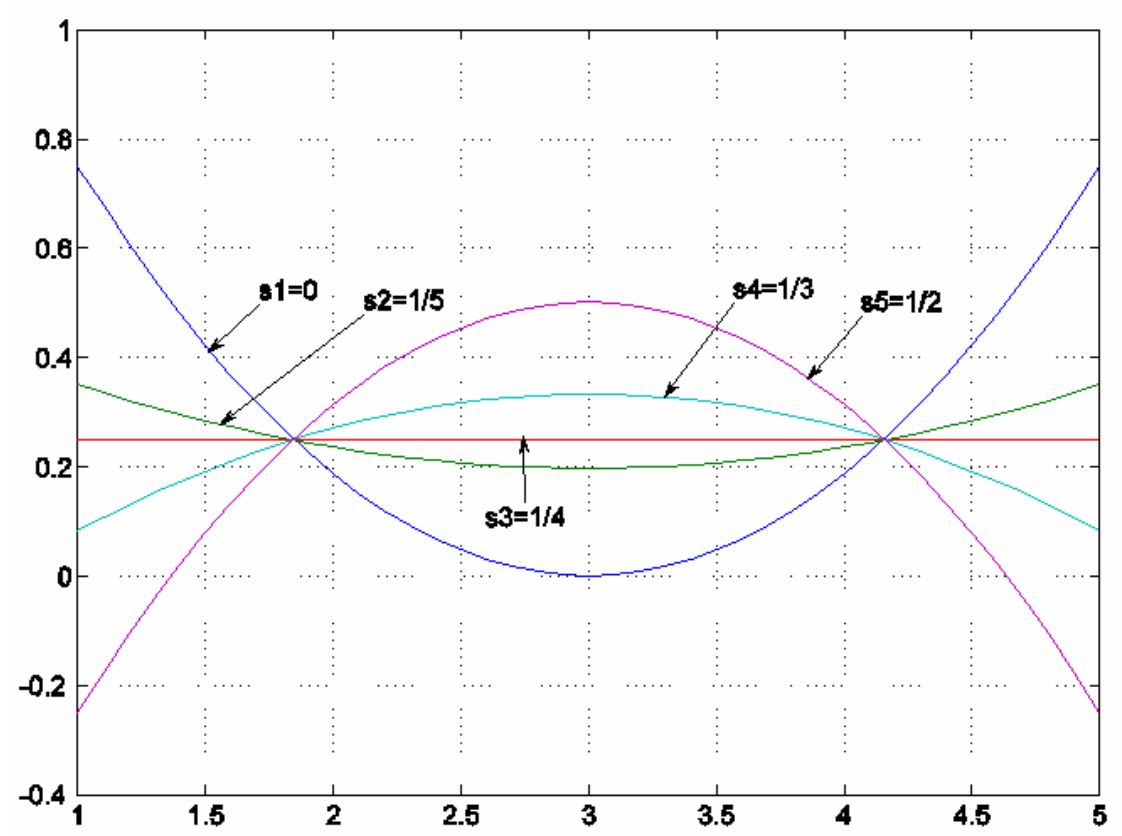

Fig. 5. Slope changes for various cubic utility functions along the assessment scale $x$

In general, utility function is non-decreasing, which means the slope at any point is non-negative, i.e., $\frac{d y}{d x}=3 a x^{2}+2 b x+c \geq 0$ in the whole definition area $\left[x_{l}, x_{h}\right]$. This gives the minimum slope $s_{\min }=0$ and the maximum slope $s_{\max }=3 / 8$ at the middle point $x_{m}$ in the above example. Also, care should be taken while using some of the curves shown in Fig. 4, as slope at some points of those curves is negative.

The six attributes listed in Table 6 are again taken as example for demonstrating the effect of using a nonlinear function as utility function. The expected utilities calculated with different slopes at the middle point are listed in Table 9. Except for $s=1 / 4$ which means that the cubic function degenerates into a linear function, the rank order of the six attributes' utilities has changed compared with the original rank order generated by the mean grade, because of the use of the nonlinear utility functions. This is a noticeable feature of using a nonlinear function as utility function. It follows that if a nonlinear function truly reflects the preferences of the decision maker on the various grades of an evaluation scale it can be used as utility function. Otherwise, a linear utility function may be the best choice in the ER approach.

Table 9 Expected utility calculated using different cubic functions

\begin{tabular}{|c|c|c|c|c|c|c|}
\hline Alternative & Mean & $s=0$ & $s=1 / 5$ & $s=1 / 4$ & $s=1 / 3$ & $s=1 / 2$ \\
\hline$a_{1}$ & 3.6 & 0.6125 & 0.6425 & 0.65 & 0.6625 & 0.6875 \\
\hline$a_{2}$ & 3.7 & 0.65625 & 0.67125 & 0.675 & 0.68125 & 0.69375 \\
\hline$a_{3}$ & 3.8 & 0.55 & 0.67 & 0.7 & 0.75 & 0.85 \\
\hline$a_{4}$ & 3.8 & 0.5875 & 0.6775 & 0.7 & 0.7375 & 0.8125 \\
\hline$a_{5}$ & 3.95 & 0.709375 & 0.731875 & 0.7375 & 0.746875 & 0.765625 \\
\hline$a_{6}$ & 4 & 0.5625 & 0.7125 & 0.75 & 0.8125 & 0.9375 \\
\hline
\end{tabular}




\section{Application Examples}

To investigate how to handle uncertainty using the methods discussed in previous sections, a case study using the general assessment framework discussed in Section 2 is conducted and reported in this Section. Due to confidentiality reasons, we have masked the original data used in our case study and only discuss the important aspects of the results. In this study, more than a hundred detailed vehicle attributes are rank-ordered using data from four different surveys. A hierarchy for assessing a detailed vehicle attribute more or less follows the hierarchy shown in Fig. 1. The IDS software is applied to assess and rank the vehicle attributes in the case study.

\subsection{Estimate a missing assessment}

To demonstrate our method for estimating missing assessments, we considered four vehicle attributes A7D01, A7D02, A7D03, and A7D04. These four vehicle attributes are the sub-attributes of the parent attribute A7D. Of these five attributes, A7D01, A7D02, A7D03 are completely assessed in the four surveys and their aggregated assessments are shown in Table 10 (see rows 5-7 of Table 10). Attribute A7D04 has some missing assessments and so does the attribute A7D. So, the problem at hand fits exactly to the description of Case 2 in Sub-section 3.3. Since the parent attribute assessments are unknown and some of the sub-attributes assessments are also unknown, Eqs. (5)-(10) are used for obtaining the estimates of value and interval (i.e., minimum and maximum values) of the unknown assessments. In estimating the missing assessments of the parent attribute $\mathrm{A} 7 \mathrm{D}$, all the sub-attributes are assumed to have equal importance as shown in Table 10. Rows 2-4 of Table 10 show the estimated value and interval for the parent attribute A7D and rows 8-10 show the estimated value and interval for the sub-attribute A7D04.

Table 10 Estimate value and estimation interval for the missing assessment

\begin{tabular}{|c|c|c|c|c|c|c|c|}
\hline Attribute & Grade 1 & Grade 2 & Grade 3 & Grade 4 & Grade 5 & $\omega$ & Remark \\
\hline A7Dmin & 0.1206 & 0.0509 & 0.2374 & 0.0509 & 0.2902 & & \multirow{3}{*}{ Predicted } \\
\hline A7Dexp & 0.1608 & 0.0678 & 0.3166 & 0.0678 & 0.3869 & & \\
\hline A7Dmax & 0.3706 & 0.3009 & 0.4874 & 0.3009 & 0.5402 & & \\
\hline A7D01 & 0.201 & 0.0663 & 0.3095 & 0.0663 & 0.3568 & 0.25 & Known \\
\hline A7D02 & 0.2060 & 0.0724 & 0.3377 & 0.0724 & 0.3116 & 0.25 & Known \\
\hline A7D03 & 0.0754 & 0.0648 & 0.3025 & 0.0648 & 0.4925 & 0.25 & Known \\
\hline A7D04min & 0 & 0 & 0 & 0 & 0 & \multirow{3}{*}{0.25} & \multirow{3}{*}{ Predicted } \\
\hline A7D04exp & 0.1608 & 0.0678 & 0.3166 & 0.0678 & 0.3869 & & \\
\hline A7D04max & 1 & 1 & 1 & 1 & 1 & & \\
\hline
\end{tabular}

\subsection{Using a quadratic mapping function}

To illustrate the effect of using a quadratic mapping function, Criteria $(1,1)$ (which is a basic criteria) in Fig. 1 is taken as an example. Suppose that all the assessments on Criteria $(1,1)$ are to be transformed to a new scale using Eq. (17) before applying the ER approach for aggregation. As a test, the middle point $t$ at $x_{m}=3$ is set to be $2,2.5,3,3.5$ and 4 respectively and for each value of $t$ the assessments on Criteria $(1,1)$ can be transformed to the new scale in an individual distribution. Based on these assessments on the new scale the new rank orders of all attributes can be obtained using the IDS software as described in Subsection 2.4. The table in Appendix A lists the top 50 ranked attributes for different values of $t$.

From the table in Appendix A, we can see that the average utility and the ranking of the top eighteen attributes are unchanged by using a quadratic mapping function for Criteria $(1,1)$. The reason is that for most of these attributes the belief degrees of assessments from Survey 1 (see Fig. 1) completely belong to the highest grade. Hence any changes in Criteria $(1,1)$ transformation would not affect these attributes. The 
ranking of the attributes in rows 19-20 (i.e., A5C and $\mathrm{A} 3 \mathrm{~A}$ ) is not affected by the transformation, but the average utility of these attributes is affected. From row 21 onwards both the ranking and average utility of the attributes is affected because of the quadratic transformation function. This study shows clearly the impact of using a quadratic mapping function on the ranking of vehicle attributes.

\subsection{Using a cubic curve as utility function}

To illustrate the effect of using a cubic utility function, the five cubic curves from $y 1$ to $y 5$ expressed in Eq. (20) and shown in Fig. 4 are used to calculate the utility values for the five grades of the desired scale. The utility values of the five grades for different cubic functions are listed in Table 11. It is obvious that at $s=$ $1 / 4$ the cubic curve degenerates into a straight line. Note that at $s=1 / 2$ the cubic curve, i.e. $y 5$ in Fig. 4, has negative slope near the two ends of the definition interval $[0,1]$.

Table 11 The utility values at every grades for given s values

\begin{tabular}{|l|c|c|c|c|c|}
\hline$u\left(H_{j}\right)$ & Grade 1 & Grade 2 & Grade 3 & Grade 4 & Grade 5 \\
\hline $\mathrm{s}=0$ & 0 & 0.4375 & 0.5 & 0.5625 & 1 \\
\hline $\mathrm{s}=1 / 5$ & 0 & 0.2875 & 0.5 & 0.7125 & 1 \\
\hline $\mathrm{s}=1 / 4$ & 0 & 0.2500 & 0.5 & 0.7500 & 1 \\
\hline $\mathrm{s}=1 / 3$ & 0 & 0.1875 & 0.5 & 0.8125 & 1 \\
\hline $\mathrm{s}=1 / 2$ & 0 & 0.0625 & 0.5 & 0.9375 & 1 \\
\hline
\end{tabular}

The table in Appendix B ranks the top 50 attributes using different cubic functions for the overall utility. Again, many of the attributes (seventeen to be specific) at the top are unaffected by the cubic utility function. The reason is that for most of these attributes the belief is associated completely to Grade 5 and so the overall utility is always one irrespective of the utility function. For the attribute in row 19 (A6I), the ranking is not affected even though the average utility is changed. From row 20 onwards, the average utility and the ranking of the attributes is affected by the cubic utility function. For the attribute "A7B02" (shown in bold in Appendix B), the average utility is reduced (and the rank order lowered) when $s<1 / 4$ and the average utility is increased (and the rank order rose) when $s>1 / 4$. On the other hand for the attribute "A3B04" (shown in bold italic in Appendix B), the observed behavior is totally opposite. This study clearly shows that using a cubic utility function affects the rank ordering of the attributes in more than one way.

\section{Concluding Remarks}

A general framework for assessing vehicle attributes using survey information was investigated. To make better use of the framework, four issues related to decision making under uncertainty are studied in the context of the ER algorithm. Applicable approaches were investigated and devised for dealing with uncertainty that may result from both original surveys and assessment aggregation. The new findings are as follows.

(1) The format of a conventional questionnaire, which only allows simple assessment on a single grade for a question, can be improved by giving respondent more choices for answering the questionnaires in a flexible yet realistic manner in order to take advantage of the features of the ER approach with the belief degree structure. Uncertainty present in a survey can be counted by means of the amount of missing evaluations divided by the total number of valid responses.

(2) For partly missing information in an assessment hierarchy, an estimate or estimation interval may be generated for each piece of the missing information. These estimates and estimation intervals can be directly used in the original ER and novel interval-based ER method $^{24-26}$ to make the final evaluation more informative and realistic.

(3) Nonlinear mapping function can be used for assessment transformation. Different functions and even different parts of a function can have significant effects in assessment transformation. However, it should be used with care as it may change the rank order of a 
group of alternatives based on the mean grades of the assessments. Only if a nonlinear function truly represents the transformation nature then it can be used as a mapping function. The choice of a transformation function is domain specific and requires expert knowledge.

(4) Similarly, a nonlinear function can be used as utility function for assessment aggregation. However, it may also change the rank order of a group of alternatives based on mean grades of the assessments. Only if a nonlinear function truly represents the decision maker's preferences then it can be used as a utility function. Based on the results of this research, it is highly recommended that a linear function be used as utility function if there is no strong evidence to support the use of any type of nonlinear functions.

Uncertainty in decision making process is complicated. In this paper, we investigated the basic approaches and their features in dealing with uncertainty possibly present in the process of vehicle evaluation. It is expected that the research findings would help make better use of survey information and deal with uncertainty in an objective and a consistent way. These can be taken as the basis for further study in this area.

\section{Acknowledgements}

The research project was sponsored by General Motors Company and conducted in close collaboration with General Motors Research and Development (GM R\&D) labs in both India and USA. The authors would like to thank GM R\&D labs for their support and all the GM personnel who have made direct contributions and provided vital support to this project.

The authors would also like to thank the reviewers and editor for their constructive suggestions for revising this manuscript.

\section{References}

1. K. S. Karam, J. S. Karam and H. H. Einstein, Decision analysis applied to tunnel exploration planning. II: Consideration of uncertainty, Journal of Construction Engineering and Management, ASCE, (2007) 354-363.

2. M. Demertzis and A. F. Tieman, Dealing with uncertainty: Robust rules in monetary policy, Scottish Journal of Political Economy, 54(2) (2007) 295-307.
3. K. E. Graves and R. Nagarajah, Uncertainty estimation using fuzzy measures for multiclass classification, IEEE Transactions on Neural Networks, 18(1) (2007) 128-140.

4. D. A. Gottlieb, T. Weiss and G. B. Chapman, The format in which uncertainty information is presented affects decision biases, Psychological Science, 18(3) (2007) 240-246.

5. D. Yu, Q. Hu and C. Wu, Uncertainty measure for fuzzy relations and their applications, Applied Soft Computing, (2007) 1135-1143.

6. C. Kahraman, A. C. Tolga, An alternative ranking approach and its usage in multi-criteria decision-making, International Journal of Computational Intelligence Systems, 2(3) (2009) $219-235$.

7. G. L. Kong, D. L. Xu and J. B. Yang, Clinical decision support systems: a review on knowledge representation and inference under uncertainties, International Journal of Computational Intelligence Systems, 1(2) (2008) 159167.

8. J.D. Power and Associates, Guide to Automotive Studies, www.jdpa.com.

9. J.D. Power Survey 2005 - How it's done, http://www.whatcar.com/news-special-report.aspx? $\mathrm{NA}=214562 \& \mathrm{EL}=3121176$, (Apr. 14, 2005).

10. Consumers Union, Road \& Trail, Consumer Reports, (2007) 48-55.

11. Consumers Union, Review of the 2006-07 models, Consumer Reports Buying Guide, (2007) 167-190.

12. Consumers Union, Rating the 2007 models, Consumer Reports Buying Guide, (2007) 157-166.

13. J. B. Yang and D. L. Xu, Knowledge based executive car evaluation using the evidential reasoning approach, in Advances in Manufacturing Technology XII (Baines, Taleb-Bendiab and Zhao eds, Professional Engineering Publishing, London, 1998) pp.741-749.

14. J. B. Yang and D. L. Xu, The IDS Multi-criteria Assessor software, Intelligent Decision System Ltd, Cheshire, UK, (2005).

15. J. B. Yang, Rule and utility based evidential reasoning approach for multiple attribute decision analysis under uncertainty, European Journal of Operational Research, 131(1) (2001) 31-61.

16. T. L. Saaty, Fundamentals of Decision Making and Priority Theory with the Analytic Hierarchy Process (RWS Publications, Pittsburgh, PA, 1994). 
17. J. B. Yang and P. Sen, A general multi-level evaluation process for hybrid MADM with uncertainty, IEEE Trans. Syst., Man, Cybern., 24(10) (1994) 1458-1473.

18. J. B. Yang and D. L. Xu, On the evidential reasoning algorithm for multiattribute decision analysis under uncertainty, IEEE Transactions on Systems, Man, and Cybernetics - Part A: Systems and Humans, 32(3) (2002) 289-304.

19. J. B. Yang and D. L. Xu, Nonlinear information aggregation via evidential reasoning in multiattribute decision analysis under uncertainty, IEEE Transactions on Systems, Man, and Cybernetics - Part A: Systems and Humans, 32(3) (2002) 376-393.

20. J. B. Yang, M. Deng, and D. L. Xu, Nonlinear regression to estimate both weights and utilities via evidential reasoning for MADM, in Proc. 5th Int. Conf. Optimization: Techniques and Applications, (Hong Kong, Dec, 2001), pp.15-17.

21. T. Kaya, Multi-attribute Evaluation of website quality in E-business using an integrated fuzzy AHPTOPSIS methodology, International Journal of Computational Intelligence Systems, 3(3) (2010) 301 - 314.

22. R. L. Keeney and H. Raiffa, Decisions with Multiple Objectives, 2nd edn. (Cambridge Univ. Press, Cambridge County U.K. 1993).

23. W. L. Winston, Operations Research-Applications and Algorithms, (Duxbury Press, Belmont, CA, 1994).

24. Y. M. Wang, J. B. Yang, D. L. Xu and K. S. Chin, The evidential reasoning approach for multiple attribute decision analysis using interval belief degrees, European Journal of Operational Research, 175(1) (2006) 35-66.

25. K. S. Chin, J. B. Yang, J. Lam and M. Guo, An evidential reasoning-interval based method for new product design assessment, IEEE transactions on Engineering Management, 56(1) (2009) 142-156.

26. Y. M. Wang, J. B. Yang and D. L. Xu, On the combination and normalization of interval-valued belief structures, Information Sciences, 177(5) (2007) 12301247. 
Appendix A Rank order contrast while using a quadratic mapping function for Criteria $(1,1)$

\begin{tabular}{|c|c|c|c|c|c|c|c|c|c|c|}
\hline Mid-point & \multicolumn{2}{|c|}{$t=2$} & \multicolumn{2}{|c|}{$\mathrm{t}=2.5$} & \multicolumn{2}{|c|}{$t=3$} & \multicolumn{2}{|c|}{$\mathrm{t}=3.5$} & \multicolumn{2}{|c|}{$t=4$} \\
\hline $\begin{array}{l}\text { Rank } \\
\text { order }\end{array}$ & $\begin{array}{c}\text { Average } \\
\text { utility }\end{array}$ & Attribute & $\begin{array}{c}\text { Average } \\
\text { utility }\end{array}$ & Attribute & $\begin{array}{c}\text { Average } \\
\text { utility }\end{array}$ & Attribute & $\begin{array}{c}\text { Average } \\
\text { utility }\end{array}$ & Attribute & $\begin{array}{l}\text { Average } \\
\text { utility }\end{array}$ & Attribute \\
\hline 1 & 1 & A3B09 & 1 & A3B09 & & A1A12 & 1 & A3B09 & 1 & A3B09 \\
\hline 2 & 1 & $\overline{\mathrm{A} 6 \mathrm{C}}$ & 1 & $\overline{A 6 C}$ & & A3A07 & 1 & $\mathrm{~A} 6 \mathrm{C}$ & 1 & A6C \\
\hline 3 & 1 & A6D03 & 1 & A6D03 & & A3B09 & 1 & A6D03 & 1 & A6D03 \\
\hline 4 & 1 & A6K06 & 1 & A6K06 & 1 & A5C04 & 1 & A6K06 & 1 & A6K06 \\
\hline 5 & 1 & A5C04 & 1 & A5C04 & 1 & A5F02 & 1 & A5C04 & 1 & A5C04 \\
\hline 6 & 1 & A6C08 & 1 & A6C08 & & A6 & 1 & A6C08 & 1 & A6C08 \\
\hline 7 & 1 & A6D04 & 1 & A6D04 & & $\mathrm{A} 6 \mathrm{C}$ & 1 & A6D04 & 1 & A6D04 \\
\hline 8 & 1 & A7B06 & 1 & A7B06 & & A6C08 & 1 & A7B06 & 1 & A7B06 \\
\hline 9 & 1 & A1A12 & 1 & A1A12 & & A6D & 1 & A1A12 & 1 & A1A12 \\
\hline 10 & 1 & A5F02 & 1 & A5F02 & & A6D01 & 1 & A5F02 & 1 & A5F02 \\
\hline 11 & 1 & A6D & 1 & A6D & & A6D02 & 1 & A6D & 1 & A6D \\
\hline 12 & 1 & A3A07 & 1 & A3A07 & & A6D03 & 1 & A3A07 & 1 & A3A07 \\
\hline 13 & 1 & A6 & 1 & A6 & & A6D04 & 1 & A6 & 1 & A6 \\
\hline 14 & 1 & A6D01 & $\overline{1}$ & A6D01 & . & A6D09 & 1 & A6D01 & 1 & A6D01 \\
\hline 15 & 1 & A6D09 & 1 & A6D09 & . & A6K06 & 1 & A6D09 & 1 & A6D09 \\
\hline 16 & 1 & A6D02 & 1 & A6D02 & & A7B06 & 1 & A6D02 & 1 & A6D02 \\
\hline 17 & 0.9872 & $\mathrm{~A} 6 \mathrm{C} 13$ & 0.9872 & $\mathrm{~A} 6 \mathrm{C} 13$ & 0.9872 & $\mathrm{A6C13}$ & 0.9872 & $\mathrm{~A} 6 \mathrm{C} 13$ & 0.9872 & A6C13 \\
\hline 18 & 0.9813 & A6I & 0.9813 & A6I & 0.9813 & A6I & 0.9813 & A6I & 0.9813 & A6I \\
\hline 19 & 0.9744 & A5C & 0.9758 & $\overline{A 5 C}$ & 0.9772 & $\overline{\mathrm{A} 5 \mathrm{C}}$ & 0.979 & $\mathrm{~A} 5 \mathrm{C}$ & 0.9807 & A5C \\
\hline 20 & 0.9392 & $\mathrm{~A} 3 \mathrm{~A}$ & 0.9421 & $\mathrm{~A} 3 \mathrm{~A}$ & 0.9451 & $\mathrm{~A} 3 \mathrm{~A}$ & 0.9492 & $\mathrm{~A} 3 \mathrm{~A}$ & 0.953 & A3A \\
\hline 21 & 0.9286 & A7B02 & 0.9286 & A7B02 & 0.9288 & A3A02 & 0.9381 & A3A02 & 0.9468 & A3A02 \\
\hline 22 & 0.9147 & A3A02 & 0.9217 & A3A02 & 0.9286 & A7B02 & 0.9319 & A6E04 & 0.9389 & A5A07 \\
\hline 23 & 0.9137 & A6E04 & 0.9195 & A6E04 & 0.9253 & A6Е04 & 0.9286 & A7B02 & 0.9384 & A3A04 \\
\hline 24 & 0.9131 & A1A06 & 0.9171 & A1A06 & 0.9212 & A1A06 & 0.9285 & A5A07 & 0.9383 & A6E04 \\
\hline 25 & 0.9102 & A6H & 0.9134 & $\mathrm{~A} 6 \mathrm{H}$ & 0.9173 & A5A07 & 0.9278 & A3A04 & 0.9355 & A6E02 \\
\hline 26 & 0.9044 & A3B01 & 0.9107 & A3B01 & 0.917 & A3B01 & 0.9272 & A1A06 & 0.9339 & A3B01 \\
\hline 27 & 0.9007 & A5A07 & 0.909 & A5A07 & 0.9165 & $\overline{\mathrm{A} 6 \mathrm{H}}$ & 0.9258 & A3B01 & 0.9328 & A1A06 \\
\hline 28 & 0.9 & A1A03 & 0.9079 & A3A04 & 0.9163 & A3A04 & 0.9253 & A6E02 & 0.9299 & A3B04 \\
\hline 29 & 0.8995 & A3A04 & 0.906 & A6E02 & 0.9142 & A6E02 & 0.9212 & $\mathrm{~A} 6 \mathrm{H}$ & 0.9289 & A5C05 \\
\hline 30 & 0.8979 & A6E02 & 0.9044 & A3B04 & 0.9113 & A3B04 & 0.9209 & A3B04 & 0.9286 & A7B02 \\
\hline 31 & 0.8975 & A3B04 & 0.9 & A1A03 & 0.9087 & A5C05 & 0.919 & A5C05 & 0.9256 & $\mathrm{~A} 6 \mathrm{H}$ \\
\hline 32 & 0.8914 & A5C05 & 0.9 & A5C05 & 0.9009 & $\overline{\mathrm{A} 1 \mathrm{~B}}$ & 0.9112 & A3B06 & 0.9224 & A3B06 \\
\hline 33 & 0.8914 & A1B & 0.8962 & A1B & 0.9 & A1A03 & 0.9068 & A1B & 0.9124 & A1B \\
\hline 34 & 0.8822 & A3B06 & 0.8907 & A3B06 & 0.8992 & A3B06 & 0.9 & A1A03 & 0.9077 & A3A01 \\
\hline 35 & 0.8781 & $\mathrm{~A} 6 \mathrm{C} 12$ & 0.8796 & $\mathrm{~A} 6 \mathrm{C} 12$ & 0.8811 & $\mathrm{~A} 6 \mathrm{C} 12$ & 0.8936 & A3A01 & 0.9 & A1A03 \\
\hline 36 & 0.875 & $\mathrm{~A} 6 \mathrm{C05}$ & 0.875 & $\mathrm{~A} 6 \mathrm{C} 05$ & 0.8787 & A3A01 & 0.8841 & $\mathrm{~A} 6 \mathrm{C} 12$ & 0.8979 & A5A05 \\
\hline 37 & 0.8547 & A3A01 & 0.8667 & A3A01 & 0.875 & A6C05 & 0.8833 & A5A05 & 0.887 & $\mathrm{~A} 6 \mathrm{C} 12$ \\
\hline 38 & 0.8436 & A5A05 & 0.8558 & A5A05 & 0.868 & A5A05 & 0.875 & A6C05 & 0.8834 & A3B08 \\
\hline 39 & 0.8381 & A2A01 & 0.8444 & A2A01 & 0.8508 & A2A01 & 0.8648 & A3B08 & 0.875 & A6C05 \\
\hline 40 & 0.8248 & A1A02 & 0.8293 & A3B08 & 0.8452 & A3B08 & 0.8595 & A2A01 & 0.8679 & A2A01 \\
\hline 41 & 0.8152 & A3B & 0.8273 & $\mathrm{~A} 1 \mathrm{~A} 02$ & 0.8297 & A1A02 & 0.8395 & A1A07 & 0.852 & A1A07 \\
\hline 42 & 0.8138 & $\mathrm{~A} 5 \mathrm{C} 02$ & 0.8213 & A3B & 0.8274 & A3B & 0.8369 & A3B & 0.8456 & A3B \\
\hline 43 & 0.8134 & A3B08 & 0.8199 & A5C02 & 0.8265 & A1A07 & 0.8351 & A5C02 & 0.8438 & A5C02 \\
\hline 44 & 0.8101 & A6A05 & 0.8178 & A1A07 & 0.826 & $\mathrm{~A} 5 \mathrm{C} 02$ & 0.8343 & A1A02 & 0.8409 & A6A05 \\
\hline 45 & 0.8088 & A1A07 & 0.816 & A6A05 & 0.8218 & A6А05 & 0.8316 & A6A05 & 0.8387 & A1A02 \\
\hline 46 & 0.8024 & A5A01 & 0.8045 & A5A01 & 0.8065 & A5A01 & 0.8139 & AX000 & 0.8302 & A3A03 \\
\hline 47 & 0.7986 & $\mathrm{~A} 1 \mathrm{~A}$ & 0.7986 & A1A & 0.8011 & AX000 & 0.8138 & A3A03 & 0.8266 & AX000 \\
\hline 48 & 0.7868 & A4A05 & 0.7911 & AX000 & 0.7986 & A1A & 0.8106 & A5A01 & 0.8185 & A3A06 \\
\hline 49 & 0.7838 & A2B01 & 0.7868 & A4A05 & 0.7964 & A3A03 & 0.8034 & A3A06 & 0.8146 & A5A01 \\
\hline 50 & 0.781 & AX000 & 0.7846 & A3A03 & 0.7892 & A1A05 & 0.7997 & A1A05 & 0.8098 & A1A05 \\
\hline
\end{tabular}


Appendix B Rank order contrast while using a cubic utility function

\begin{tabular}{|c|c|c|c|c|c|c|c|c|c|c|}
\hline M.P.- & \multicolumn{2}{|c|}{$s=0$} & \multicolumn{2}{|c|}{$s=1 / 5$} & \multicolumn{2}{|c|}{$s=1 / 4$} & \multicolumn{2}{|c|}{$s=1 / 3$} & \multicolumn{2}{|c|}{$s=1 / 2$} \\
\hline $\begin{array}{l}\text { Rank } \\
\text { order }\end{array}$ & $\begin{array}{c}\text { Average } \\
\text { utility }\end{array}$ & Attribute & $\begin{array}{c}\text { Average } \\
\text { utility }\end{array}$ & Attribute & $\begin{array}{c}\text { Average } \\
\text { utility }\end{array}$ & Attribute & $\begin{array}{c}\text { Average } \\
\text { utility }\end{array}$ & Attribute & $\begin{array}{c}\text { Average } \\
\text { utility }\end{array}$ & Attribute \\
\hline 1 & 1 & A3B09 & 1 & АЗВ09 & 1 & A3B09 & 1 & A3B09 & 1 & A3B09 \\
\hline 2 & 1 & A6C & 1 & $\mathrm{~A} 6 \mathrm{C}$ & 1 & A6C & 1 & $A 6 C$ & 1 & $A 6 C$ \\
\hline 3 & 1 & A6D03 & 1 & A6D03 & 1 & A6D03 & 1 & A6D03 & & A6D03 \\
\hline 4 & 1 & A6K06 & 1 & A6K06 & & A6K06 & 1 & A6K06 & & A6K06 \\
\hline 5 & 1 & $\mathrm{~A} 5 \mathrm{CO4}$ & 1 & $\mathrm{~A} 5 \mathrm{CO4}$ & 1 & $\mathrm{~A} 5 \mathrm{CO4}$ & 1 & $\mathrm{~A} 5 \mathrm{CO4}$ & & $\mathrm{A} 5 \mathrm{CO4}$ \\
\hline 6 & 1 & A6C08 & 1 & $A 6 C 08$ & 1 & A6C08 & 1 & $\mathrm{~A} 6 \mathrm{C08}$ & & A6C08 \\
\hline 7 & 1 & A6D04 & $\overline{1}$ & A6D04 & 1 & A6D04 & 1 & A6D04 & & A6D04 \\
\hline 8 & 1 & A7B06 & 1 & A7B06 & 1 & A7B06 & 1 & A7B06 & & A7B06 \\
\hline 9 & 1 & A1A12 & 1 & A1A12 & 1 & A1A12 & 1 & A1A12 & & A1A12 \\
\hline 10 & 1 & A5F02 & 1 & A5F02 & & A5F02 & 1 & A5F02 & 1 & A5F02 \\
\hline 11 & 1 & $A 6 D$ & 1 & $A 6 D$ & & $A 6 D$ & 1 & $A 6 D$ & & $A 6 D$ \\
\hline 12 & 1 & A3A07 & 1 & A3A07 & & A3A07 & 1 & A3A07 & & A3A07 \\
\hline 13 & 1 & A6 & 1 & $A 6$ & 1 & A6 & 1 & $A 6$ & & $A 6$ \\
\hline 14 & 1 & A6D01 & 1 & A6D01 & 1 & A6D01 & 1 & A6D01 & 1 & A6D01 \\
\hline 15 & 1 & A6D09 & $\overline{1}$ & A6D09 & 1 & A6D09 & 1 & A6D09 & & A6D09 \\
\hline 16 & 1 & A6D02 & 1 & A6D02 & & A6D02 & 1 & A6D02 & 1 & A6D02 \\
\hline 17 & 0.9872 & $\mathrm{~A} 6 \mathrm{C} 13$ & 0.9872 & $\mathrm{~A} 6 \mathrm{C} 13$ & 0.9872 & $\mathrm{~A} 6 \mathrm{C} 13$ & 0.9872 & $\mathrm{~A} 6 \mathrm{C} 13$ & 0.9872 & $\mathrm{~A} 6 \mathrm{C} 13$ \\
\hline 18 & 0.9774 & A6I & 0.9805 & $A 6 I$ & 0.9813 & A6I & 0.9826 & A6I & 0.9852 & A6I \\
\hline 19 & 0.9743 & $A 5 C$ & 0.9766 & $A 5 C$ & 0.9772 & $A 5 C$ & 0.9782 & $A 5 C$ & 0.9821 & A7B02 \\
\hline 20 & 0.9391 & A3A & 0.9439 & A3A & 0.9451 & A3A & 0.9471 & A3A & 0.9801 & A5C \\
\hline 21 & 0.9193 & A3A02 & 0.9269 & A3A02 & 0.9288 & A3A02 & 0.9464 & A7B02 & 0.9688 & A6C05 \\
\hline 22 & 0.9165 & A6E04 & 0.9235 & A6E04 & 0.9286 & A7B02 & 0.9319 & A3A02 & 0.9511 & A3A \\
\hline 23 & 0.9111 & $\mathrm{~A} 6 \mathrm{H}$ & 0.9189 & A1A06 & 0.9253 & A6E04 & 0.9282 & A6E04 & 0.9383 & A3A02 \\
\hline 24 & 0.9098 & A1A06 & 0.9179 & A7B02 & 0.9212 & A1A06 & 0.925 & A1A06 & 0.9375 & $\mathrm{~A} 6 \mathrm{H} 12$ \\
\hline 25 & 0.9089 & A5A07 & 0.9156 & A5A07 & 0.9173 & A5A07 & 0.9201 & A5A07 & 0.9341 & A6E04 \\
\hline 26 & 0.9083 & A3B01 & 0.9154 & $\mathrm{~A} 6 \mathrm{H}$ & 0.917 & A3B01 & 0.92 & A3B01 & 0.9327 & A1A06 \\
\hline 27 & 0.9057 & A3A04 & 0.9153 & A3B01 & 0.9165 & $\mathrm{~A} 6 \mathrm{H}$ & 0.9198 & A3A04 & 0.9268 & A3A04 \\
\hline 28 & 0.9047 & A6E02 & 0.9142 & A3А04 & 0.9163 & A3A04 & 0.9183 & $\mathrm{~A} 6 \mathrm{H}$ & 0.9258 & A3B01 \\
\hline 29 & 0.9026 & A3B04 & 0.9123 & A6E02 & 0.9142 & A6E02 & 0.9174 & A6E02 & 0.9257 & A5A07 \\
\hline 30 & 0.9 & A1A03 & 0.9096 & A3B04 & 0.9113 & A3B04 & 0.9142 & A3B04 & 0.9237 & A6E02 \\
\hline 31 & 0.8976 & A1B & 0.9064 & A5C05 & 0.9087 & A5C05 & 0.9125 & A5C05 & 0.9219 & $\mathrm{~A} 6 \mathrm{H}$ \\
\hline 32 & 0.8972 & A5C05 & 0.9003 & A1B & 0.9009 & A1B & 0.9063 & A6C05 & 0.9202 & A5C05 \\
\hline 33 & 0.89 & A3B06 & 0.9 & A1A03 & 0.9 & A1A03 & 0.9023 & A3B06 & 0.9201 & A3B04 \\
\hline 34 & 0.8778 & $\mathrm{~A} 6 \mathrm{C} 12$ & 0.8974 & A3B06 & 0.8992 & A3B06 & 0.902 & A1B & 0.9085 & A3B06 \\
\hline 35 & 0.875 & A7B02 & 0.8805 & A6C12 & 0.8811 & $\mathrm{~A} 6 \mathrm{C} 12$ & 0.9 & A1A03 & 0.9043 & A1B \\
\hline 36 & 0.8644 & A3A01 & 0.8759 & A3A01 & 0.8787 & A3A01 & 0.8835 & A3A01 & 0.9 & A1A03 \\
\hline 37 & 0.855 & A5A05 & 0.8654 & A5A05 & 0.875 & A6C05 & 0.8822 & $\mathrm{~A} 6 \mathrm{C} 12$ & 0.893 & A3A01 \\
\hline 38 & 0.8459 & $\mathrm{~A} 1 \mathrm{~A} 02$ & 0.8563 & $A 6 C 05$ & 0.868 & A5A05 & 0.8723 & A5A05 & 0.8845 & $\mathrm{~A} 6 \mathrm{C} 12$ \\
\hline 39 & 0.8447 & $\mathrm{~A} 5 \mathrm{C} 02$ & 0.8488 & A2A01 & 0.8508 & $\mathrm{~A} 2 \mathrm{~A} 01$ & 0.8541 & $\mathrm{~A} 2 \mathrm{~A} 01$ & 0.8812 & A1A07 \\
\hline 40 & 0.8409 & $\mathrm{~A} 2 \mathrm{~A} 01$ & 0.8431 & A3В08 & 0.8452 & A3B08 & 0.8487 & A3B08 & 0.881 & A5A05 \\
\hline 41 & 0.8346 & A3B08 & 0.833 & A1A02 & 0.8297 & A1A02 & 0.8447 & A1A07 & 0.8607 & $\mathrm{~A} 2 \mathrm{~A} 01$ \\
\hline 42 & 0.8164 & A3B & 0.8297 & $\mathrm{~A} 5 \mathrm{C} 02$ & 0.8274 & A3B & 0.8311 & A3B & 0.859 & $A X 000$ \\
\hline 43 & 0.8138 & A6A05 & 0.8252 & A3B & 0.8265 & A1A07 & 0.8245 & A6A05 & 0.8558 & A3B08 \\
\hline 44 & 0.8051 & A3A06 & 0.8202 & A6A05 & 0.826 & A5C02 & 0.8244 & A1A02 & 0.8384 & A3B \\
\hline 45 & 0.7876 & A1A & 0.8156 & A1A07 & 0.8218 & A6A05 & 0.8204 & $A \times 000$ & 0.8306 & A5A01 \\
\hline 46 & 0.7861 & A4A05 & 0.8017 & A5A01 & 0.8065 & A5A01 & 0.8198 & $\mathrm{~A} 5 \mathrm{C} 02$ & 0.8298 & A6A05 \\
\hline 47 & 0.7837 & A2B01 & 0.7964 & A1A & 0.8011 & $A \times 000$ & 0.8146 & A5A01 & 0.8136 & A1A02 \\
\hline 48 & 0.7832 & A3A03 & 0.7937 & A3A03 & 0.7986 & A1A & 0.8125 & $\mathrm{~A} 6 \mathrm{H} 12$ & 0.8096 & A3A03 \\
\hline 49 & 0.7824 & A5A01 & 0.7909 & A3A06 & 0.7964 & A3A03 & 0.8022 & A1A & 0.8095 & A1A \\
\hline 50 & 0.7813 & $\mathrm{~A} 6 \mathrm{CO5}$ & 0.7895 & AX000 & 0.7892 & A1A05 & 0.8008 & A3A03 & 0.8073 & $\mathrm{~A} 5 \mathrm{C} 02$ \\
\hline
\end{tabular}

